\title{
Quantum Ito's Formula and Stochastic Evolutions *
}

\author{
R. L. Hudson ${ }^{1}$ and K. R. Parthasarathy ${ }^{2}$ \\ 1 Mathematics Department, University of Nottingham, University Park, Nottingham \\ NG7 2RD, England \\ 2 Indian Statistical Institute, 7, S.J.S. Sansanwal Marg, New Delhi 110 016, India
}

\begin{abstract}
Using only the Boson canonical commutation relations and the Riemann-Lebesgue integral we construct a simple theory of stochastic integrals and differentials with respect to the basic field operator processes. This leads to a noncommutative Ito product formula, a realisation of the classical Poisson process in Fock space which gives a noncommutative central limit theorem, the construction of solutions of certain noncommutative stochastic differential equations, and finally to the integration of certain irreversible equations of motion governed by semigroups of completely positive maps. The classical Ito product formula for stochastic differentials with respect to Brownian motion and the Poisson process is a special case.
\end{abstract}

\section{Introduction}

We construct a quantum mechanical generalisation of the Ito-Doob theory of mean-square stochastic integration and an associated Ito product formula in which Brownian motion is replaced by the pair of operator processes $\left(A_{f}(t): t \geqq 0\right)$, $\left(A_{g}^{\dagger}(t): t \geqq 0\right)$, where $A_{f}(t)=a\left(f \chi_{[0, t]}\right)$, and $A_{g}^{\dagger}(t)=a^{\dagger}\left(g \chi_{[0, t]}\right)$ are annihilation and creation operators in the Boson Fock space $\Gamma(\mathfrak{h})$ over $\mathfrak{h}=L^{2}[0, \infty) \otimes \mathfrak{f}, \mathfrak{f}$ being a Hilbert space with inner product $\langle\cdot, \cdot\rangle_{\mathfrak{t}}$, and the Poisson process is replaced by what we call a gauge process $\left(\Lambda_{I I}(t): t \geqq 0\right)$, where $\pi$ is a locally bounded self adjoint operator valued map from $[0, \infty)$ to $B(\mathfrak{t})$ and $\Lambda_{\pi}(t)$ is the differential second quantisation of $I \otimes \Pi(t)$. This leads to a stochastic calculus which is in some respects simpler and more natural than the classical theory, which is contained as a special case.

* Parts of this work were completed while the first author was a Royal Society-Indian National Science Academy Exchange Visitor to the Indian Statistical Institute, New Delhi, and visiting the University of Texas supported in part by NSF grant PHY81-07381, and part while the second author was visiting the Mathematics Research Centre of the University of Warwick 
The germ of our method is the identity

$$
\left\langle\psi(h), \psi\left(h^{\prime}\right)\right\rangle_{\Gamma(\mathbf{h})}=\exp \int_{0}^{\infty}\left\langle h(s), h^{\prime}(s)\right\rangle_{\mathrm{f}} d s
$$

for the exponential vectors or coherent states corresponding to $h, h^{\prime} \in \mathfrak{h}$, together with the eigenrelation

$$
A_{f}(t) \psi(h)=\left\{\int_{0}^{\infty}\langle f(s), h(s)\rangle_{\mathrm{r}} d s\right\} \psi(h),
$$

which has the differential form

$$
d A_{f} \psi(h)=\langle f(t), h(t)\rangle_{\mathrm{t}} d t \psi(h) .
$$

This together with the adjoint relationship between $a$ and $a^{\dagger}$ and the field commutation relations enables us to express the matrix elements between exponential vectors of integrals against $d \Lambda_{\pi}, d A_{f}, d A_{g}^{\dagger}$ as integrals against $d t$ (Theorem 4.1). For simple integrands the Ito product formula is then a consequence of ordinary differential calculus and elementary formulae for matrix elements between exponential vectors of products of the $A_{f}(t), A_{g}^{\dagger}(t)$, and $\Lambda_{I I}(t)$ (Theorem 4.3). The same theorem then provides estimates permitting the extension of the integral to locally square integrable processes with a corresponding extension of the product formula.

Specialising to the case $\mathfrak{F}=\mathbb{C}, f=g=\Pi \equiv 1$ and denoting the basic processes in this case by $A(t), A^{\dagger}(t)$, and $\Lambda(t)$, we are able to realise the Poisson process with intensity $l$ as a commuting self-adjoint operator valued process in the Fock space admitting the differential representation

$$
d P_{l}=d \Lambda+\sqrt{l}\left(d A+d A^{\dagger}\right)+l d t .
$$

The classical formula $\left(d P_{l}\right)^{2}=d P_{l}$, and a noncommutative central limit theorem for the Poisson process are immediate corollaries. The gauge process $\Lambda(t)$ may be interpreted as the Poisson process of zero intensity.

We exploit the stochastic calculus developed here by constructing processes $(U(t): t \geqq 0)$ which may be regarded as stochastically autonomous quantum evolutions in that they satisfy stochastic differential equations of the form

$$
d U=U\left(L_{1} d \Lambda+L_{2} d A+L_{3} d A^{\dagger}+L_{4} d t\right), \quad U(0)=I,
$$

characterised by a quadruple of infinitesimal generators generalising the conventional Hamiltonian. When these are bounded operators, we find necessary and sufficient conditions on them for the process to be unitary (Theorem 7.1). Each such unitary process generates in a natural way a uniformly continuous semigroup of completely positive maps, indicating that the formalism developed here permits the integration of irreversible equations of motion described by such semigroups, as studied by Lindblad [10] and by Gorini et al. [4]. We emphasise that we do not find a group dilation of such semigroups; the unitary operators $U(t)$ do not satisfy the group law $U(s) U(t)=U(s+t)$.

Heuristic and partial accounts of the theory now presented rigorously were given in $[6,7]$. The recent paper [3] provides what is, in effect, the Fermion analog 
of classical Brownian motion regarded as a subtheory of the Boson quantum Brownian motion used here, insofar as the basic process used is the combination $A+A^{\dagger}$ of the Fermion annihilation and creation processes $A$ and $A^{\dagger}$. Much of the theory developed here transforms to Fermions with $A$ and $A^{\dagger}$ treated independently [2].

We use the following notations and conventions. Hilbert space inner products are linear on the right. $\mathfrak{D}(T)$ denotes the domain of the operator $T$, which is said to be on $\mathfrak{D}(T)$. The Hilbert space adjoint of $T$ is denoted $T^{*}$. Operators $T$ and $T^{\dagger}$ on $\mathfrak{D}(T)$ and $\mathfrak{D}\left(T^{\dagger}\right)$ are mutually adjoint if for all $\psi \in \mathfrak{D}(T)$ and $\varphi \in \mathfrak{D}\left(T^{\dagger}\right),\langle T \psi, \varphi\rangle$ $=\left\langle\psi, T^{\dagger} \varphi\right\rangle$. The algebraic tensor product of vector spaces $\mathfrak{R}$ and $\mathfrak{H}$ is denoted by $\mathfrak{S} \otimes \mathfrak{H}$; if they are Hilbert spaces, $\mathfrak{i} \otimes \mathfrak{H}$ denotes the Hilbert space completion. If $T$ is an operator on $\mathfrak{D}$ then the operator on $\mathfrak{D} \otimes \mathfrak{H}$ whose action on proudct vectors is $u \otimes \psi \mapsto T u \otimes \psi$ is called the ampliation of $\bar{T}$ to $\mathfrak{D} \otimes \mathfrak{H} \cdot \chi_{S}$ denotes the indicator function of a set $S$ taking value 1 on $S$ and 0 on its complement. $\int^{t} f$ denotes the Riemann-Lebesgue integral of $f$ over the interval $[s, t] . B(\mathfrak{h})$ denotes the *-algebra of bounded operators on the Hilbert space $\mathfrak{h}$.

\section{The Weyl Representation}

Let $\mathfrak{h}$ be a Hilbert space and let $\Gamma(\mathfrak{h})$ be the boson Fock space over $\mathfrak{h}$. For each $f \in \mathfrak{h}$, let $\psi(f)$ be the corresponding exponential vector

$$
\psi(f)=\left(1, f, \ldots,(n !)^{-1 / 2} f \otimes \ldots \otimes f, \ldots\right),
$$

so that $\psi(0)$ is the Fock vacuum $\psi_{0}$.

Let $\mathscr{U}(\mathfrak{h})$ denote the group of unitary operators on $\mathfrak{h}$ with the strong topology and let $\mathscr{G}=\mathfrak{h}(\mathfrak{U}(\mathfrak{h})$ be the semidirect product of the "translation group" $\mathfrak{h}$ with the "rotation group" $\mathscr{U}(\mathfrak{h})$, that is the Euclidean group of $\mathfrak{h}$. For each $(f, U) \in \mathscr{G}$ we define the unitary operator $W(f, U)$ by its action on exponential vectors

$$
W(f, U) \psi(g)=\exp \left(-1 / 2\|f\|^{2}-\langle f, U g\rangle\right) \psi(U g+f)
$$

for $g \in \mathfrak{h}$. Then for $f, g \in \mathfrak{h}, U, V \in \mathscr{U}(\mathfrak{h})$,

$$
W(f, U) W(g, V)=\exp (-i \operatorname{Im}\langle f, U g\rangle) W(f+U g, U V) .
$$

Thus $W$ is a projective unitary representation of $\mathscr{G}$ with multiplier

$$
\sigma((f, U),(g, V))=\exp (-i \operatorname{Im}\langle f, U g\rangle),
$$

which we call the Weyl representation of $\mathscr{G}$.

We require the infinitesimal form of the Weyl representation. For $f \in \mathfrak{h}$ and $T$ $\in B(\mathfrak{h})$, define operators $a(f), a^{\dagger}(f)$, and $\lambda(T)$ on the dense domain $\mathfrak{E}$ of finite linear combinations of exponential vectors in $\Gamma(\mathrm{h})$ by

$$
\begin{gathered}
a(f) \psi(g)=\langle f, g\rangle \psi(g), \quad a^{\dagger}(f) \psi(g)=\left.\frac{d}{d \varepsilon} \psi(g+\varepsilon f)\right|_{\varepsilon=0}, \\
\lambda(T) \psi(g)=\left.\frac{d}{d \varepsilon} \psi\left(e^{\varepsilon T} f\right)\right|_{\varepsilon=0} .
\end{gathered}
$$


Since the exponential vectors are linearly independent these are well defined. Moreover for arbitrary $f, g, h \in \mathfrak{h}$ and $T \in B(\mathfrak{h})$,

$$
\left.\begin{array}{rl}
\langle\psi(f), a(h) \psi(g)\rangle & =\langle h, g\rangle\langle\psi(f), \psi(g)\rangle, \\
\left\langle\psi(f), a^{\dagger}(h) \psi(g)\right\rangle & =\langle f, h\rangle\langle\psi(f), \psi(g)\rangle, \\
\langle\psi(f), \lambda(T) \psi(g)\rangle & =\langle f, T g\rangle\langle\psi(f), \psi(g)\rangle,
\end{array}\right\}
$$

showing in particular that $a(h)$ and $a^{\dagger}(h)$ are mutually adjoint on $\mathbb{E}$, as are $\lambda(T)$ and $\lambda\left(T^{*}\right)$. Furthermore for arbitrary $e, f, g, h \in \mathfrak{h}$ and $S, T \in B(\mathfrak{h})$,

$$
\begin{aligned}
\langle a(e) \psi(f), a(h) \psi(g)\rangle & =\langle f, e\rangle\langle h, g\rangle\langle\psi(f), \psi(g)\rangle, \\
\left\langle a^{\dagger}(e) \psi(f), a^{\dagger}(h) \psi(g)\right\rangle & =\{\langle f, h\rangle\langle e, g\rangle+\langle e, h\rangle\}\langle\psi(f), \psi(g)\rangle, \\
\langle\lambda(S) \psi(f), \lambda(T) \psi(g)\rangle & =\{\langle S f, T g\rangle+\langle S f, g\rangle\langle f, T g\rangle\}\langle\psi(f), \psi(g)\rangle, \\
\langle a(h) \psi(f), \lambda(T) \psi(g)\rangle & =\langle f, h\rangle\langle f, T g\rangle\langle\psi(f), \psi(g)\rangle, \\
\left\langle a^{\dagger}(h) \psi(f), \lambda(T) \psi(g)\right\rangle & =\{\langle h, g\rangle\langle f, T g\rangle+\langle h, T g\rangle\}\langle\psi(f), \psi(g)\rangle, \\
\left\langle a^{\dagger}(e) \psi(f), a(h) \psi(g)\right\rangle & =\langle h, g\rangle\langle e, g\rangle\langle\psi(f), \psi(g)\rangle .
\end{aligned}
$$

We also define mutually adjoint operators $e^{a(f)}$ and $e^{a^{\dagger}(f)}$ on $\mathbb{E}$ by

$$
e^{a(f)} \psi(g)=e^{\langle f, g\rangle} \psi(g), \quad e^{a^{\dagger}(f)} \psi(g)=\psi(g+f) .
$$

Then as an operator on $\mathfrak{E}, W(f, U)$ admits the factorisation

$$
W(f, U)=e^{-\frac{1}{2}\|f\|^{2}} e^{a^{\dagger}(f)} W(0, U) e^{a(-U f)} .
$$

\section{Operator-valued Processes}

Let $\mathfrak{f}$ be a Hilbert space. We denote by $\mathfrak{h}, \mathfrak{h}_{t}$, and $\mathfrak{h}^{t}$ the Hilbert spaces $L_{\mathfrak{f}}^{2}[0, \infty)$, $L_{\mathfrak{f}}^{2}[0, t]$, and $L_{\mathfrak{f}}^{2}(t, \infty)$ of square-integrable measurable vector-valued functions taking values in $\mathfrak{f}$, and by $\mathfrak{H}, \mathfrak{H}_{t}$, and $\mathfrak{H}^{t}$ their respective Fock spaces. Corresponding to the natural decomposition $\mathfrak{h}=\mathfrak{h}_{t} \oplus \mathfrak{h}^{t}$, we make the identification $\mathfrak{H}=\mathfrak{H}_{t} \otimes \mathfrak{H}^{t}$ in which for each exponential vector $\psi(f), f \in h, \psi(f)=\psi\left(f_{t}\right) \otimes \psi\left(f^{t}\right)$, where $f_{t}$ and $f^{t}$ are the components of $f$ in $\mathfrak{h}_{t}$ and $\mathfrak{h}^{t}$, respectively. We denote by $\mathfrak{E}_{t}$ and $\mathfrak{E}^{t}$ the dense subspaces of $\mathfrak{H}_{t}$ and $\mathfrak{H}^{t}$ spanned by the exponential vectors.

The operator-valued processes which concern us live in the tensor product $\tilde{\mathfrak{H}}=\mathfrak{H} \otimes \mathfrak{G}$ of $\mathfrak{H}$ with a Hilbert space $\mathfrak{R}$ called the initial space. We write $\widetilde{\mathfrak{H}}_{t}=\mathfrak{H} \otimes \mathfrak{H}_{t}$ so that $\widetilde{\mathfrak{H}}=\widetilde{\mathfrak{H}}_{t} \otimes \mathfrak{H}^{t}$.

The following proposition is a straightforward generalisation of the well known fact that the exponential vectors are linearly independent.

Proposition 3.1. Let $u, v, \ldots$ be nonzero elements of $\mathfrak{R}$, and let $f, g, \ldots$ be distinct elements of $\mathfrak{h}$. Then the vectors $u \otimes \psi(f), v \otimes \psi(g), \ldots$ are linearly independent in $\widetilde{\mathfrak{H}}$.

Let $S \subset \mathfrak{h}$ be a real linear manifold closed under all the projections $f \mapsto f_{t}, t \geqq 0$ and such that $S+i S$ is dense. We call such $S$ an admissible subspace and denote by $\mathfrak{G}$ and $\mathfrak{S}_{t}$ the dense subspaces of $\mathfrak{H}$ and $\mathfrak{H}_{t}$ spanned by the exponential vectors $\psi(f)$ and $\psi\left(f_{t}\right)$, respectively, with $f \in S$. Let $\mathfrak{D}$ be a linear manifold in $\mathfrak{R}$, and let $\alpha:(u, f) \mapsto \alpha(u, f)$ be a map from $\mathfrak{D} \times S$ to $\widetilde{\mathfrak{H}}$ which is linear in $u$ for each fixed $f \in S$. 
Then it follows from Proposition 3.1 that there is a unique operator in $\tilde{\mathfrak{H}}$ with domain $\mathfrak{D} \otimes \mathbb{S}$ (which is dense if $\mathfrak{D}$ is dense in $\mathfrak{R}$ ) mapping each $u \otimes \psi(f), f \in S$ to $\alpha(u, f)$.

Definition 3.1. Let $S \subset \mathfrak{h}$ be an admissible subspace and $\mathfrak{D}$ a linear manifold in $\mathfrak{\Re}$. Let $F=(F(t): t \geqq 0)$ be a family of operators in $\widetilde{\mathfrak{H}}$ such that for arbitrary $t>0, F(t)$ is the ampliation to $\mathfrak{D} \otimes \mathfrak{S}_{t} \otimes \mathfrak{S}^{t}$ of an operator in $\widetilde{\mathfrak{H}}_{t}$ with domain $\mathfrak{D} \otimes \mathfrak{S}_{t}$. Then $F$ is called an adapted process based on $(\mathfrak{D}, S)$.

A family $F=(F(t): t \geqq 0)$ of bounded operators on $\tilde{\mathfrak{H}}$ determines an adapted process based on $(\mathfrak{D}, S)$ by restricting the domain of each $F(t)$ to $\mathfrak{D} \otimes \mathfrak{S}_{t} \otimes \mathfrak{H}^{t}$ if and only if each $F(t)$ belongs to the von Neumann algebra $B\left(\widetilde{\mathfrak{H}}_{t}\right) \otimes I$. Such a process will be said to be bounded and to be unitary if each of the original operators is unitary.

The adapted processes based on $(\mathfrak{D}, S)$ form a complex vector space which we denote by $\mathfrak{A}(\mathfrak{D}, S)$.

Definition 3.3. An element $F \in \mathfrak{A}(\mathfrak{D}, S)$ is said to be simple if there exists an increasing sequence $t_{n}, n=0,1, \ldots$ with $t_{0}=0$ and $t_{n} \underset{n}{\longrightarrow} \infty$ such that

$$
F=\sum_{n=0}^{\infty} F_{n} \chi_{\left[t_{n}, t_{n+1}\right)},
$$

where $F_{n}=F\left(t_{n}\right)$, to be continuous if for each $u \in \mathfrak{D}$ and $f \in S$ the map $t \rightarrow F(t) u \otimes \psi(f)$ is strongly continuous from $[0, \infty)$ to $\widetilde{\mathfrak{H}}$, and to be locally square integrable if each such map is strongly measurable and satisfies $\int_{0}^{t}\|F(s) u \otimes \psi(f)\|^{2} d s<\infty$ for all $t>0$.

${ }_{0}$ We denote by $\mathfrak{A}_{0}(\mathfrak{D}, S), \mathfrak{A}_{c}(\mathfrak{D}, S)$, and $L^{2}(\mathfrak{D}, S)$ the subspaces of $\mathfrak{\mathfrak { l }}(\mathfrak{D}, S)$ of simple, continuous and locally square integrable processes. Clearly

$$
\mathfrak{A}_{c}(\mathfrak{D}, S) \subset L^{2}(\mathfrak{D}, S) \text {. }
$$

We thank P. L. Muthuramalingam for assistance in the proof of the following proposition.

Proposition 3.2. Let $F \in L^{2}(\mathcal{D}, S)$. Then there exists a sequence $F^{(n)}, \mathrm{n}=1,2, \ldots$ of simple processes such for each $t>0, u \in \mathfrak{D}$, and $f \in S$,

$$
\lim _{n} \int_{0}^{t}\left\|\left(F(s)-F^{(n)}(s)\right) u \otimes \psi(f)\right\|^{2} d s=0 .
$$

Proof. Denote by $\varphi_{n}$ the probability density $\varphi_{n}(t)=n \chi_{[0,1 / n]}(t)$, and set

$$
F_{n}(t) u \otimes \psi(f)=\int_{0}^{t} \varphi_{n}(t-s)(F(s) u \otimes \psi(f)) d s=n \int_{t-1 / n}^{t} F(s) u \otimes \psi(f) d s,
$$

where we set $F(s) \equiv 0$ for $s<0$. Then $F_{n}$ is a continuous process and

$$
\begin{aligned}
\left\|\left(F_{n}(t)-F(t)\right) u \otimes \psi(f)\right\|^{2} & =\left\|\int_{0}^{\infty} \varphi_{n}(s)(F(t-s)-F(t)) u \otimes \psi(f) d s\right\|^{2} \\
& \leqq \int_{0}^{\infty}\|(F(t-s)-F(t)) u \otimes \varphi(f)\|^{2} \varphi_{n}(s) d s \\
& \leqq \sup \left\{\|(F(t-s)-F(t)) u \otimes \psi(f)\|^{2}: 0 \leqq s \leqq 1 / n\right\},
\end{aligned}
$$


where we use the fact that $\varphi_{n}$ is a probability density and the Hilbert space Jensen's inequality. Hence for arbitrary $T>0$,

$$
\int_{0}^{T}\left\|\left(F_{n}(t)-F(t)\right) u \otimes \psi(f)\right\|^{2} d t \leqq \sup _{0 \leqq s \leqq 1 / n} \int_{0}^{T}\|(F(t-s)-F(t)) u \otimes \psi(f)\|^{2} d t .
$$

By Lebesgue's theorem for vector-valued functions we conclude that

$$
\lim _{n} \int_{0}^{T}\left\|\left(F_{n}(t)-F(t)\right) u \otimes \psi(f)\right\|^{2} d t=0
$$

Now define $F^{(n)}$ by $F^{(n)}(t)=F_{n}\left(m 2^{-n}\right)$ if $m 2^{-n} \leqq t<(m+1) 2^{-n}, m=0,1, \ldots$. Then $F^{(n)} \in \mathfrak{A}_{0}(\mathfrak{D}, S)$. Fix $t$ in the interval $\left[m 2^{-n},(m+1) 2^{-n}\right)$. Since $\varphi_{n}\left(m 2^{-n}-s\right)=0$ for $s>m 2^{-n}$ we have

$$
\begin{aligned}
& \left\|\left(F^{(n)}(t)-F_{n}(t)\right) u \otimes \psi(f)\right\|^{2} \\
& \quad=\left\|\int_{0}^{m 2-n} \varphi_{n}\left(m 2^{-n}-s\right)(F(s) u \otimes \psi(f)) d s-\int_{0}^{t} \varphi_{n}(t-s)(F(s) u \otimes \psi(f)) d s\right\|^{2} \\
& \quad=\left\|\int_{0}^{t}\left(\varphi_{n}\left(m 2^{-n}-s\right)-\varphi_{n}(t-s)\right)(F(s) u \otimes \psi(f)) d s\right\|^{2} \\
& \quad \leqq\left(\int_{0}^{t}\left|\varphi_{n}\left(m 2^{-n}-s\right)-\varphi_{n}(t-s)\right|^{2} d s\right)\left(\int_{0}^{t}\|F(s) u \otimes \psi(f)\|^{2} d s\right) \\
& \quad \leqq\left(\int_{0}^{T}\|F(s) u \otimes \psi(f)\|^{2} d s\right) n^{2} 2^{-n}
\end{aligned}
$$

for all $t \in[0, T]$. Together with (3.2) this implies that

$$
\lim _{n} \int_{0}^{T}\left\|\left(F^{(n)}(t)-F(t)\right) u \otimes \psi(f)\right\|^{2} d t=0
$$

\section{Stochastic Integrals and Ito's Formula}

Let $f$ and $g$ be elements of the space $L_{\mathbf{t}}^{\infty}$, loc $[0, \infty)$ of locally bounded measurable vector-valued functions from $[0, \infty)$ into $\mathfrak{k}$, and let $\Pi$ be an element of the space $L_{B(\mathfrak{t})}^{\infty, \text { loc }}[0, \infty)$ of locally bounded measurable functions from $[0, \infty)$ into the Banach space $B(\mathfrak{f})$. Then for each $t \geqq 0, f_{t}=f \chi_{[0, t]}$ and $g_{t}=g \chi_{[0, t]} \in \mathfrak{h}$, and $\Pi_{t}=\Pi \chi_{[0, t]}$ may be regarded as an element of $B(\mathfrak{h})$ acting pointwise on $\mathfrak{h}$. We may thus construct the operators

$$
A_{f}(t)=a\left(f_{t}\right), \quad A_{g}^{\dagger}(t)=a^{\dagger}\left(g_{t}\right), \quad \Lambda_{\Pi}(t)=\lambda\left(\Pi_{t}\right) .
$$

We identify these operators with their ampliations in $\widetilde{\mathfrak{H}}=\mathfrak{R} \otimes \mathfrak{H}$; they then form adapted processes based on $(\mathfrak{S}, \mathfrak{h})$ called the annihilation, creation, and gauge processes, of strengths $f, g$, and $\Pi$, respectively. They form the integrators with respect to which we shall develop stochastic integrals.

Proposition 4.1. $A_{f}, A_{g}^{\dagger}$, and $\Lambda_{\Pi}$ are continuous, hence locally square integrable. 
Proof. Using the first three of relations (2.4) it is easily seen that

$$
\begin{aligned}
&\left\|\left(A_{f}(t)-A_{f}(s)\right) u \otimes \psi(h)\right\|^{2}=\left|\int_{s}^{t}\langle f(r), h(r)\rangle_{\mathrm{f}} d r\right|^{2}\|u \otimes \psi(h)\|^{2}, \\
&\left\|\left(A_{g}^{\dagger}(t)-A_{g}^{\dagger}(s)\right) u \otimes \psi(h)\right\|^{2}=\left\{\left|\int_{s}^{t}\langle g(r), h(r)\rangle_{\mathfrak{t}} d r\right|^{2}+\int_{s}^{t}\|g(r)\|_{\mathfrak{t}}^{2} d r\right\}\|u \otimes \psi(h)\|^{2}, \\
&\left\|\left(\Lambda_{\Pi}(t)-\Lambda_{\Pi}(s)\right) u \otimes \psi(h)\right\|^{2}=\left\{\int_{s}^{t}\|\Pi(r) h(r)\|_{\mathfrak{f}}^{2} d r+\left(\int_{s}^{t}\langle\Pi(r) h(r), h(r)\rangle_{\mathfrak{f}} d r\right)^{2}\right\} \\
& \cdot\|u \otimes \psi(h)\|^{2},
\end{aligned}
$$

for arbitrary $t>s \geqq 0, u \in \mathfrak{R}$, and $h \in \mathfrak{h}$, from which the continuity is clear.

Definition 4.1. Let $E, F, G, H \in \mathfrak{A}_{0}(\mathfrak{D}, S)$, so that we may write

$$
\begin{aligned}
E=\sum_{n=0}^{\infty} E_{n} \chi_{\left[t_{n}, t_{n+1}\right)}, & F=\sum_{n=0}^{\infty} F_{n} \chi_{\left[t_{n}, t_{n+1}\right)}, \\
G=\sum_{n=0}^{\infty} G_{n} \chi_{\left[t_{n}, t_{n+1}\right)}, & H=\sum_{n=0}^{\infty} H_{n} \chi_{\left[t_{n}, t_{n+1}\right)},
\end{aligned}
$$

where $0=t_{0}<t_{1}<t_{2}<\ldots<t_{n} \longrightarrow \infty$. The family of operators $M=(M(t): t \geqq 0)$, with domain $\mathfrak{D} \otimes S$ defined by

$$
\begin{gathered}
M(0)=0 \\
M(t)=M\left(t_{n}\right)+E_{n}\left(\Lambda_{I I}(t)-\Lambda_{I I}\left(t_{n}\right)\right)+F_{n}\left(A_{f}(t)-A_{f}\left(t_{n}\right)\right) \\
+G_{n}\left(A_{g}^{\dagger}(t)-A_{g}^{\dagger}\left(t_{n}\right)\right)+H_{n}\left(t-t_{n}\right)
\end{gathered}
$$

for $t_{n}<t \leqq t_{n+1}$, is called the stochastic integral of $(E, F, G, H)$ with respect to $\Lambda_{I I}, A_{f}, A_{g}$ and Lebesgue measure, and denoted by

$$
M(t)=\int_{0}^{t}\left(E d \Lambda_{\Pi I}+F d A_{f}+G d A_{g}^{\dagger}+H d S\right) .
$$

Clearly $M \in \mathfrak{U}(\mathfrak{D}, S)$ and depends linearly on $(E, F, G, H)$.

Theorem 4.1. Let $E, F, G, H \in \mathfrak{A}_{0}(\mathfrak{D}, S)$, and let $M$ be their stochastic integral. Then for arbitrary $u \in \mathfrak{S}, e \in \mathfrak{h}, v \in \mathfrak{D}, h \in S$, and $t \geqq 0$,

$$
\begin{aligned}
\langle u \otimes \psi(e), M(t) v \otimes \psi(h)\rangle= & \int_{0}^{t}\left\langle u \otimes \psi(e),\left\{\langle e(s), \Pi(s) h(s)\rangle_{\mathrm{t}} E(s)+\langle f(s), h(s)\rangle_{\mathrm{t}} F(s)\right.\right. \\
& \left.\left.+\langle e(s), g(s)\rangle_{\mathrm{f}} G(s)+H(s)\right\} v \otimes \psi(h)\right\rangle d s .
\end{aligned}
$$

Proof. Assume that $E, F, G, H$ are given by (4.1) and $M(t)$ by (4.2). Noting first that (4.4) holds when $t=0$, we prove it for $t \in\left(t_{n}, t_{n+1}\right]$ by induction on $n$. By (4.2) we have for such $t$

$$
\begin{aligned}
\langle u \otimes \psi(e), M(t) v \otimes \psi(h)\rangle= & \left\langle u \otimes \psi(e), M\left(t_{n}\right) v \otimes \psi(h)\right\rangle \\
& +\left\langle u \otimes \psi(e), E_{n}\left(\Lambda_{\Pi}(t)-\Lambda_{I}\left(t_{n}\right)\right) v \otimes \psi(h)\right\rangle \\
& +\left\langle u \otimes \psi(e), F_{n}\left(A_{f}(t)-A_{f}\left(t_{n}\right)\right) v \otimes \psi(h)\right\rangle \\
& +\left\langle u \otimes \psi(e), G_{n}\left(A_{g}^{\dagger}(t)-A_{g}^{\dagger}\left(t_{n}\right)\right) v \otimes \psi(h)\right\rangle \\
& +\left\langle u \otimes \psi(e), H_{n}\left(t-t_{n}\right) v \otimes \psi(h)\right\rangle .
\end{aligned}
$$


Using the decomposition $\widetilde{\mathfrak{H}}=\widetilde{\mathfrak{H}}_{t_{n}} \otimes \mathfrak{H}^{t_{n}}$, we note that, in the second term on the right of (4.4) $E_{n}$ is the ampliation of an operator in $\widetilde{\mathfrak{H}}_{t_{n}}$, whereas $\Lambda_{\Pi}(t)-\Lambda_{\Pi}\left(t_{n}\right)$ is the ampliation of the operator $\lambda\left(\Pi_{\left(t_{n}, t\right]}\right)$, which we regard as an operator in $\mathfrak{H}^{t_{n}}$. Factorising the vectors $u \otimes \psi(e)$ and $v \otimes \psi(h)$ as $u \otimes \psi\left(e_{t_{n}}\right) \otimes \psi\left(e^{t_{n}}\right)$ and $v \otimes \psi\left(h_{t_{n}}\right) \otimes \psi\left(h^{t_{n}}\right)$, and applying the third identity of $(2.3)$, we write this term as

$$
\begin{aligned}
\int_{t_{n}}^{t}\left\langle u \otimes \psi(e),\langle e(s), \Pi(s) h(s)\rangle_{\mathfrak{f}} E_{n} v \otimes \psi(h)\right\rangle d s \\
\quad=\int_{t_{n}}^{t}\left\langle u \otimes \psi(e),\langle e(s), \Pi(s) h(s)\rangle_{\mathfrak{f}} E(s) v \otimes \psi(h)\right\rangle d s,
\end{aligned}
$$

since $E(s)=E\left(t_{n}\right)$ for $t_{n} \leqq s<t$. Similar arguments using the remaining identities of (2.3) enable us to equate the third and fourth terms on the right of (4.4) to

$$
\begin{gathered}
\int_{t_{n}}^{t}\left\langle u \otimes \psi(e),\langle f(s), h(s)\rangle_{\mathfrak{f}} F(s) v \otimes \psi(h)\right\rangle d s, \\
\int_{t_{n}}^{t}\left\langle u \otimes \psi(e),\langle e(s), g(s)\rangle_{\mathfrak{f}} G(s) v \otimes \psi(h)\right\rangle d s,
\end{gathered}
$$

respectively. Writing the final term as $\int^{t}\langle u \otimes \psi(e), H(s) v \otimes \psi(h)\rangle d s$, and using the inductive hypothesis to write $\left\langle u \otimes \psi(e), M\left(t_{n}\right) v \otimes \psi(h)\right\rangle$ in the form (4.4) completes the proof.

Theorem 4.2. Let $E, F, G, H \in \mathfrak{U}_{0}(\mathfrak{D}, S)$, and let $M$ be their stochastic integral. Let $0 \leqq s<t, \varphi \in \widetilde{\mathfrak{H}}_{s}, e \in \mathfrak{h}, h \in S$, and $v \in \mathfrak{D}$. Then

$$
\begin{aligned}
& \left\langle\varphi \otimes \psi\left(e^{s}\right),(M(t)-M(s)) v \otimes \psi(h)\right\rangle \\
& =\int_{s}^{t}\left\langle\varphi \otimes \psi ( e ^ { s } ) \left\{\langle e(r), \Pi(r) h(r)\rangle_{\mathfrak{f}} E(r)\right.\right. \\
& \left.\left.\quad+\langle f(r), h(r)\rangle_{\mathfrak{f}} F(r)+\langle e(r), g(r)\rangle_{\mathfrak{f}} G(r)+H(r)\right\} v \otimes \psi(h)\right\rangle d r .
\end{aligned}
$$

Proof. If $\varphi$ is of form $u \otimes \psi\left(d_{s}\right)$ with $u \in \mathfrak{R}, d \in \mathfrak{h}$ the theorem follows from Theorem 4.1 in which we replace $e$ by $d \chi_{[0, s]}+e \chi_{(s, \infty)}$, and subtract from (4.4) the corresponding identity with $t$ replaced by $s$. But vectors of this form are total in $\widetilde{\mathfrak{H}}_{s}$. Hence a limiting argument gives the general case.

The next theorem is crucial. It is essentially Ito's formula for simple integrands. We shall use it to derive estimates permitting the extension of stochastic integration to locally square integrable processes. The theorem, together with its two predecessors, remains true for such processes as we shall see.

Theorem 4.3. Let

$$
\begin{aligned}
M(t) & =\int_{0}^{t}\left(E d \Lambda_{\Pi}+F d A_{f}+G d A_{g}^{\dagger}+H d S\right), \\
M^{\prime}(t) & =\int_{0}^{t}\left(E^{\prime} d \Lambda_{\Pi^{\prime}}+F^{\prime} d A_{f^{\prime}}+G^{\prime} d A_{g^{\prime}}^{\dagger}+H^{\prime} d s\right),
\end{aligned}
$$


where $E, F, G, H \in \mathfrak{A}_{0}(\mathfrak{D}, S), E^{\prime}, F^{\prime}, G^{\prime}, H^{\prime} \in \mathfrak{A}_{0}\left(\mathfrak{D}^{\prime}, S^{\prime}\right)$. Then for all $u \in \mathfrak{D}, u^{\prime} \in \mathfrak{D}^{\prime}, h$ $\in S, h^{\prime} \in S^{\prime}$, and $t \geqq 0$,

$$
\begin{aligned}
&\left\langle M(t) u \otimes \psi(h), M^{\prime}(t) u^{\prime} \otimes \psi\left(h^{\prime}\right)\right\rangle \\
&=\int_{0}^{t}\left\{\left\langleM(s) u \otimes \psi(h),\left[\left\langle h(s), \Pi^{\prime}(s) h^{\prime}(s)\right\rangle_{\mathfrak{t}} E^{\prime}(s)\right.\right.\right. \\
&\left.\left.+\left\langle f^{\prime}(s), h^{\prime}(s)\right\rangle_{\mathfrak{f}} F^{\prime}(s)+\left\langle h(s), g^{\prime}(s)\right\rangle_{\mathfrak{t}} G^{\prime}(s)+H^{\prime}(s)\right] u^{\prime} \otimes \psi\left(h^{\prime}\right)\right\rangle \\
&+\left\langle\left[\left\langle h^{\prime}(s), \Pi(s) h(s)\right\rangle_{\mathrm{f}} E(s)+\langle f(s), h(s)\rangle_{\mathrm{f}} F(s)\right.\right. \\
&\left.\left.+\left\langle h^{\prime}(s), g(s)\right\rangle_{\mathfrak{f}} G(s)+H(s)\right] u \otimes \psi(h), M^{\prime}(s) u^{\prime} \otimes \psi\left(h^{\prime}\right)\right\rangle \\
&+\langle\Pi(s) h(s) \otimes E(s) u \otimes \psi(h)+g(s) \otimes G(s) u \otimes \psi(h), \\
&\left.\left.\Pi^{\prime}(s) h^{\prime}(s) \otimes E^{\prime}(s) u^{\prime} \otimes \psi^{\prime}(h)+g^{\prime}(s) \otimes G^{\prime}(s) u^{\prime} \otimes \psi\left(h^{\prime}\right)\right\rangle_{\mathfrak{k} \otimes \mathfrak{H}}\right\} d s .
\end{aligned}
$$

Proof. Assume $E, F, G, H$ and similarly $E^{\prime}, F^{\prime}, G^{\prime}, H^{\prime}$ given by (4.1). Equation (4.7) holds for $t=0$; we prove inductively that it holds for $t \in\left(t_{n}, t_{n+1}\right]$. For such $t$ from (4.2)

$$
\begin{aligned}
&\left\langle M(t) u \otimes \psi(h), M^{\prime}(t) u^{\prime} \otimes \psi\left(h^{\prime}\right)\right\rangle \\
&=\left\langle M\left(t_{n}\right)+E_{n}\left(\Lambda_{\Pi I}(t)-\Lambda_{\Pi}\left(t_{n}\right)\right)+F_{n}\left(A_{f}(t)-A_{f}\left(t_{n}\right)\right)\right. \\
&\left.\quad+G_{n}\left(A_{g}^{\dagger}(t)-A_{g}^{\dagger}\left(t_{n}\right)\right)+H_{n}\left(t-t_{n}\right) u \otimes \psi h\right), \\
& \quad M^{\prime}\left(t_{n}\right)+E_{n}^{\prime}\left(\Lambda_{\Pi^{\prime}}(t)-\Lambda_{\Pi^{\prime}}\left(t_{n}\right)\right)+F_{n}^{\prime}\left(A_{f^{\prime}}(t)-A_{f^{\prime}}\left(t_{n}\right)\right) \\
&+\left.G_{n}^{\prime}\left(A_{g^{\prime}}^{\dagger}(t)-A_{g^{\prime}}^{\dagger}\left(t_{n}\right)\right)+H_{n}^{\prime}\left(t-t_{n}\right) u^{\prime} \otimes \psi\left(h^{\prime}\right)\right\rangle .
\end{aligned}
$$

Set $\tilde{\mathfrak{Y}}=\tilde{\mathfrak{H}}_{t_{n}} \otimes \mathfrak{H}^{t_{n}} \cdot M\left(t_{n}\right), M^{\prime}\left(t_{n}\right), E_{n}, F_{n}, G_{n}, H_{n}, E_{n}^{\prime}, F_{n}^{\prime}, G_{n}^{\prime}$, and $H_{n}^{\prime}$ are ampliations of operators in $\tilde{\mathfrak{H}_{t}}$, on the other hand $\Lambda_{I I}(t)-\Lambda_{I I}\left(t_{n}\right), A_{f}(t)-A_{f}\left(t_{n}\right), A_{g}^{\dagger}(t)-A_{g}^{\dagger}\left(t_{n}\right)$, $\Lambda_{\Pi I^{\prime}}(t)-\Lambda_{\Pi I^{\prime}}\left(t_{n}\right), \quad A_{f^{\prime}}(t)-A_{f^{\prime}}\left(t_{n}\right)$, and $A_{g^{\prime}}^{\dagger}(t)-A_{g^{\prime}}^{\dagger}\left(t_{n}\right)$ are the ampliations of $\lambda\left(\Pi \chi_{\left(t_{n}, t\right)}\right), a\left(f \chi_{\left(t_{n}, t\right]}\right), a^{\dagger}\left(g \chi_{\left(t_{n}, t\right]}\right), \lambda\left(\Pi^{\prime} \chi_{\left(t_{n}, t\right]}\right), a\left(f^{\prime} \chi_{\left(t_{n}, t\right]}\right)$, and $a^{\dagger}\left(g^{\prime} \chi_{\left(t_{n}, t\right]}\right)$, respectively regarded as operators in the space $\mathfrak{H}^{t_{n}}$. Factorising the vectors $u \otimes \psi(h)$ and $u^{\prime} \otimes \psi\left(h^{\prime}\right)$ as $u \otimes \psi\left(h_{t_{n}}\right) \otimes \psi\left(h^{t_{n}}\right)$ and $u^{\prime} \otimes \psi\left(h_{t_{n}}^{\prime}\right) \otimes \psi\left(h^{\prime t_{n}}\right)$, and using the identities (2.3) and (2.4), we obtain

$$
\begin{aligned}
&\left\langle M(t) u \otimes \psi(h), M^{\prime}(t) u^{\prime} \otimes \psi\left(h^{\prime}\right)\right\rangle \\
&=\left\langle\left\{ M\left(t_{n}\right)+E_{n} \int_{t_{n}}^{t}\left\langle h^{\prime}(s), \Pi(s) h(s)\right\rangle_{\mathrm{f}} d s+F_{n} \int_{t_{n}}^{t}\langle f(s), h(s)\rangle_{\mathrm{f}} d s\right.\right. \\
&+\left.G \int_{t_{n}}^{t}\left\langle h^{\prime}(s), g(s)\right\rangle_{\mathrm{r}} d s+H_{n}\left(t-t_{n}\right)\right\} u \otimes \psi(h), \\
&\left\{M^{\prime}\left(t_{n}\right)+E_{n}^{\prime} \int_{t_{n}}^{t}\left\langle h(s), \Pi^{\prime}(s) h^{\prime}(s)\right\rangle_{\mathrm{f}} d s+F_{n}^{\prime} \int_{t_{n}}^{t}\left\langle f^{\prime}(s), h^{\prime}(s)\right\rangle_{\mathrm{f}} d s\right. \\
&+\left.\left.G_{n}^{\prime} \int_{t_{n}}^{t}\left\langle h(s), g^{\prime}(s)\right\rangle_{\mathrm{f}} d s+H_{n}^{\prime}\left(t-t_{n}\right)\right\} u^{\prime} \otimes \psi\left(h^{\prime}\right)\right\rangle \\
&+ \int_{t_{n}}^{t}\left\langle\Pi(s) h(s), \Pi^{\prime}(s) h^{\prime}(s)\right\rangle_{\mathrm{r}} d s\left\langle E_{n} u \otimes \psi(h), E_{n}^{\prime} u^{\prime} \otimes \psi\left(h^{\prime}\right)\right\rangle \\
&+ \int_{t_{n}}^{t}\left\langle\Pi(s) h(s), g^{\prime}(s)\right\rangle_{\mathrm{r}} d s\left\langle E_{n} u \otimes \psi(h), G_{n}^{\prime} u^{\prime} \otimes \psi\left(h^{\prime}\right)\right\rangle
\end{aligned}
$$




$$
\begin{aligned}
& +\int_{t_{n}}^{n}\left\langle g(s), \Pi^{\prime}(s) h^{\prime}(s)\right\rangle_{\mathfrak{h}} d s\left\langle G_{n} u \otimes \psi(h), E_{n}^{\prime} u^{\prime} \otimes \psi\left(h^{\prime}\right)\right\rangle \\
& +\int_{t_{n}}^{t}\left\langle g(s), g^{\prime}(s)\right\rangle_{\mathrm{f}} d s\left\langle G_{n} u \otimes \psi(h), G_{n}^{\prime} u^{\prime} \otimes \psi\left(h^{\prime}\right)\right\rangle .
\end{aligned}
$$

We now differentiate with respect to $t$. The derivative of the first term on the right of (4.8) is the sum of two terms obtained by differentiating separately the two sides of the inner product. These differentiations result in vectors of form $\varphi^{\prime} \otimes \varphi\left(h^{t_{n}}\right)$, $\varphi^{\prime} \otimes \psi\left(h^{\prime t_{n}}\right), \varphi, \varphi^{\prime} \in \mathfrak{S}_{t_{n}}$. On the opposite side of the inner product we bring the coefficients $E_{n}, F_{n}, G_{n}$ or $E_{n}^{\prime}, F_{n}^{\prime}, G_{n}^{\prime}$ inside the integrals as $E(s), F(s), G(s)$ or $E^{\prime}(s)$, $F^{\prime}(s), G^{\prime}(s)$, and replace $H_{n}$ or $H_{n}^{\prime}$ by $\int_{t_{n}}^{t} H(s) d s$ or $\int_{t_{n}}^{t} H^{\prime}(s) d s$. We may now use Theorem 4.2, in which $s$ is taken to be $t_{n}$, to equate the derivative of the first term on the right of (4.8) to the sum of the first two terms of the integrand on the right of (4.7). A similar argument shows that the derivative of the remaining terms on the right of (4.8) is equal to the remaining term in the integrand of (4.7). By the inductive hypothesis we may assume that (4.7) holds when $t=t_{n}$, and so the theorem follows from the fundamental theorem of calculus.

Corollary 1. Suppose that $S$ consists of locally bounded functions, so that

$$
\begin{aligned}
\alpha(T)= & \sup _{0 \leqq s \leqq T} \max \left\{\left|\langle h(s), \Pi(s) h(s)\rangle_{\mathfrak{f}},\right|\langle f(s), h(s)\rangle_{\mathfrak{f}} \mid,\right. \\
& \left.\left|\langle h(s), g(s)\rangle_{\mathfrak{f}}\right|,\|\Pi(s) h(s)\|_{\mathfrak{t}}^{2},\|g(s)\|_{\mathfrak{f}}^{2}\right\}
\end{aligned}
$$

is finite for each $T>0$. Then for $T>0$ and $0 \leqq t \leqq T$,

$$
\begin{aligned}
\|M(t) u \otimes \psi(h)\|^{2} \leqq & 6 \alpha(T)^{2} \int_{0}^{T} e^{t-s}\left\{\|E(s) u \otimes \psi(h)\|^{2}+\|F(s) u \otimes \psi(h)\|^{2}\right. \\
& \left.+\|G(s) u \otimes \psi(h)\|^{2}+\|H(s) u \otimes \psi(h)\|^{2}\right\} d s .
\end{aligned}
$$

Proof. Setting $\left(E^{\prime}, F^{\prime}, G^{\prime}, H^{\prime}\right)=(E, F, G, H), u^{\prime}=u$, and $h^{\prime}=h$ in the theorem gives

$$
\begin{aligned}
\|M(t) u \otimes \psi(h)\|^{2}= & \int_{0}^{t}\left\{2 \operatorname { R e } \left\langleM(s) u \otimes \psi(h),\left[\langle h(s), \Pi(s) h(s)\rangle_{\mathfrak{t}} E(s)\right.\right.\right. \\
& \left.\left.+\langle f(s), h(s)\rangle_{\mathfrak{f}} F(s)+\langle h(s), g(s)\rangle_{\mathfrak{t}} G(s)+H(s)\right] u \otimes \psi(h)\right\rangle \\
& \left.+\|I I(s) h(s) \otimes E(s) u \otimes \psi(h)+g(s) \otimes G(s) u \otimes \psi(h)\|^{2}\right\} d s .
\end{aligned}
$$

Using the inequalities for Hilbert space vectors $\xi, \eta, \xi_{1}, \xi_{2}, \ldots, \xi_{n}$,

$$
2|\langle\xi, \eta\rangle| \leqq\|\xi\|^{2}+\|\eta\|^{2}, \quad\left\|\xi_{1}+\xi_{2}+\ldots+\xi_{n}\right\|^{2} \leqq n\left(\left\|\xi_{1}\right\|^{2}+\ldots+\left\|\xi_{n}\right\|^{2}\right),
$$

we find that the integrand in (4.10) is bounded above by

$$
\begin{aligned}
\|M(s) u \otimes \psi(h)\|^{2} & +6 \alpha(T)^{2}\left\{\|E(s) u \otimes \psi(h)\|^{2}+\|F(s) u \otimes \psi(h)\|^{2}\right. \\
& \left.+\|G(s) u \otimes \psi(h)\|^{2}+\|H(s) u \otimes \psi(h)\|^{2}\right\},
\end{aligned}
$$

and hence, differentiating, that

$$
\begin{aligned}
\frac{d}{d s}\|M(s) u \otimes \psi(h)\|^{2} \leqq & \|M(s) u \otimes \psi(h)\|^{2}+6 \alpha(T)^{2}\left\{\|E(s) u \otimes \psi(h)\|^{2}+\|F(s) u \otimes \psi(h)\|^{2}\right. \\
& \left.+\|G(s) u \otimes \psi(h)\|^{2}+\|H(s) u \otimes \psi(h)\|^{2}\right\} .
\end{aligned}
$$


Multiplying by the integrating factor $e^{-s}$ and integrating over the interval $[0, t]$ we obtain (4.9).

Corollary 2. Under the hypothesis of Corollary 1, for $0 \leqq s \leqq t<T$,

$$
\begin{aligned}
\|(M(t)-M(s)) u \otimes \psi(h)\|^{2}= & 6 \alpha(T)^{2} \int_{s}^{t} e^{t-r}\left\{\|E(r) u \otimes \psi(h)\|^{2}+\|F(r) u \otimes \psi(h)\|^{2}\right. \\
& \left.+\|G(r) u \otimes \psi(h)\|^{2}+\|H(r) u \otimes \psi(h)\|^{2}\right\} d r .
\end{aligned}
$$

In particular $M$ is continuous and thus $M \in L^{2}(\mathfrak{D}, S)$.

Proof. Replace $E, F, G, H$ in Corollary 1 by $E \chi_{[s, \infty)}, F \chi_{[s, \infty)}, G \chi_{[s, \infty)}, H \chi_{[s, \infty)}$.

We are now ready to extend the stochastic integral to integrands in $L^{2}(\mathfrak{D}, S)$, under the assumption that $S$ consists of locally bounded functions. Let $E, F, G, H$ $\in L^{2}(\mathfrak{D}, S)$. By Proposition 3.2 there exist $E^{(n)}, F^{(n)}, G^{(n)}, H^{(n)}, n=1,2, \ldots$ in $\mathfrak{A}_{0}(\mathfrak{D}, S)$ such that for all $u \in \mathfrak{D}, h \in S$, and $t>0$,

$$
\begin{aligned}
& \lim _{n} \int_{0}^{t}\left\{\left\|\left(E(s)-E^{(n)}(s)\right) u \otimes \psi(h)\right\|^{2}+\left\|\left(F(s)-F^{(n)}(s)\right) u \otimes \psi(h)\right\|^{2}\right. \\
& \left.\left.\quad+\| G(s)-G^{(n)}(s)\right) u \otimes \psi(h)\left\|^{2}+\right\|\left(H(s)-H^{(n)}(s)\right) u \otimes \psi(h) \|^{2}\right\} d s=0 .
\end{aligned}
$$

Now let $M^{(n)}, n=1,2, \ldots$ be the stochastic integrals

$$
M^{(n)}(t)=\int_{0}^{t}\left(E^{(n)} d \Lambda_{\Pi}+F^{(n)} d A_{f}+G^{(n)} d A_{g}^{\dagger}+H^{(n)} d s\right) .
$$

Applying Corollary 1 to the differences $M^{(n)}-M^{(m)}$, we see that the sequence $M^{(n)}(t) u \otimes \psi(h)$ converges in $\widetilde{\mathfrak{H}}$ for each $t \geqq 0, u \in \mathfrak{D}$, and $h \in S$, and that the limit does not depend on the choice of $\left(E^{(n)}, F^{(n)}, G^{(n)}, H^{(n)}\right)$. The limit is clearly linear in $u$, and thus defines an operator with domain $\mathfrak{D} \otimes \mathbb{S}$, which can be extended uniquely as the ampliation to $\mathfrak{D} \otimes \mathfrak{S}_{t} \otimes \mathfrak{S}^{t}$ of an operator in $\mathfrak{H}_{t}$ with domain $\mathfrak{D} \otimes \mathfrak{S}_{t}$. We denote the extended operator by $M(t)$. Then $M=(M(t): t \geqq 0)$ is an adapted process we define to be the stochastic integral,

$$
M(t)=\int_{0}^{t}\left(E d \Lambda_{\Pi}+F d A_{f}+G d A_{g}^{\dagger}+H d s\right) .
$$

It is clear that $M$ depends linearly on $(E, F, G, H)$.

Theorem 4.4. Provided that $S, S^{\prime}$ consist of locally bounded functions, Theorems 4.1-4.3 and Corollaries 1 and 2 remain true for locally square integrable integrands.

Proof. The inequalities (4.9) and (4.11) follow from the definition of $M$ and from continuity of the norm. From (4.11) it follows that for each $u \in \mathfrak{D}$ and $h \in S$, $t \mapsto M(t) u \otimes \psi(h)$ is strongly continuous and hence bounded on finite intervals. We may thus pass to the limit of simple approximations in (4.7), as well as (4.4) and (4.6).

We use the differential notation

$$
d M=E d \Lambda_{\Pi}+F d A_{f}+G d A_{g}^{\dagger}+H d t
$$


to describe the situation that $M$ is a process in $L^{2}(\mathcal{D}, S)$ (where $S$ consists of locally bounded functions) such that for all $t \geqq 0$,

$$
M(t)-M(0)=\int_{0}^{t}\left(E d \Lambda_{\Pi}+F d A_{f}+G d A_{g}^{\dagger}+H d s\right),
$$

where $E, F, G, H \in L^{2}(\mathfrak{D}, S)$.

To avoid difficulties with existence of adjoints and products we now consider bounded processes. For a fixed admissible subspace $S$ consisting of locally bounded functions, we denote by $\mathfrak{C}(S)$ the set of all processes $M \in \mathfrak{A}(\mathfrak{R}, S)$ satisfying (4.10) for some $E, F, G, H \in \mathfrak{U}_{c}(\mathfrak{\Re}, S)$, and some $f, g \in L_{\mathfrak{f}}^{\infty, l^{\circ}}[0, \infty)$ and $\Pi \in L_{B(\mathfrak{t})}^{\infty, \text { loc }}[0, \infty)$ such that for all $t>0$,

$$
\sup _{0 \leqq s \leqq t} \max \{\|M(s)\|,\|E(s)\|,\|F(s)\|,\|G(s)\|,\|H(s)\|\}<\infty .
$$

Theorem (7.1) below shows that $\mathfrak{C}(S)$ is by no means empty. We denote by $\mathfrak{M}(S)$ the linear span of $\mathfrak{C}(S)$. We can now state Ito's formula.

Theorem 4.5. $\mathfrak{M}(S)$ is an algebra, in which multiplication is given by

$$
d\left(M M^{\prime}\right)=M d M^{\prime}+(d M) M^{\prime}+d M d M^{\prime},
$$

for $M, M^{\prime} \in \mathbb{C}(S)$, where for $M$ satisfying (4.12), $(d M) M^{\prime}$ is given by

$$
(d M) M^{\prime}=E M^{\prime} d \Lambda_{I I}+F M^{\prime} d A_{f}+G M^{\prime} d A_{g}^{\dagger}+H M^{\prime} d t,
$$

that is, the basic differentials $d \Lambda_{I}, d A_{f}, d A_{g}^{\dagger}$, and $d t$ commute with the adapted process $M^{\prime}$, and $d M d M^{\prime}$ is evaluated by combining this with extension by bilinearity of the multiplication rules

\begin{tabular}{c|cccc} 
& $d \Lambda_{\Pi \Pi^{\prime}}$ & $d A_{f^{\prime}}$ & $d A_{g^{\prime}}^{\dagger}$ & $d t$ \\
\hline$d \Lambda_{\Pi}$ & $d \Lambda_{\Pi \Pi^{\prime}}$ & 0 & $d A_{\Pi g^{\prime}}^{\dagger}$ & 0 \\
$d A_{f}$ & $d A_{\Pi \Pi^{\prime} f}^{\dagger}$ & 0 & $\left\langle f(t), g^{\prime}(t)\right\rangle d t$ & 0 \\
$d A_{g}^{\dagger}$ & 0 & 0 & 0 & 0 \\
$d t$ & 0 & 0 & 0 & 0
\end{tabular}

Proof. We may assume without loss of generality that $M(0)=M^{\prime}(0)=0$, since multiplication by constant processes follows the rules stated. We note in view of (4.12),

$$
d M^{\dagger}=E^{\dagger} d \Lambda_{I I^{\dagger}}+G^{\dagger} d A_{g}+F^{\dagger} d A_{f}^{\dagger}+H^{\dagger} d t .
$$

Replacing $M$ by $M^{\dagger}$ in (4.7) we find that, for $u, u^{\prime} \in \mathfrak{R}, h, h^{\prime} \in S$ and $t>0$,

$$
\begin{aligned}
&\langle u \otimes\left.\psi(h), M(t) M^{\prime}(t) u^{\prime} \otimes \psi\left(h^{\prime}\right)\right\rangle=\left\langle M^{\dagger}(t) u \otimes \psi(h), M^{\prime}(t) u^{\prime} \otimes \psi\left(h^{\prime}\right)\right\rangle \\
&=\int_{0}^{t}\left\{\left\langleM^{\dagger}(s) u \otimes \psi(h),\left[\left\langle h(s), \Pi^{\prime}(s) h^{\prime}(s)\right\rangle_{\mathfrak{t}} E^{\prime}(s)\right.\right.\right. \\
&\left.\left.\quad+\left\langle f^{\prime}(s), h^{\prime}(s)\right\rangle_{\mathfrak{t}} F^{\prime}(s)+\left\langle h(s), g^{\prime}(s)\right\rangle_{\mathfrak{t}} G^{\prime}(s)+H^{\prime}(s)\right] u^{\prime} \otimes \psi\left(h^{\prime}\right)\right\rangle \\
& \quad+\left\langle\left[h^{\prime}(s), \Pi^{\dagger}(s) h(s)\right\rangle_{\mathfrak{t}} E^{\dagger}(s)+\langle g(s), h(s)\rangle_{\mathfrak{f}} G^{\dagger}(s)\right. \\
&\left.\left.\quad+\left\langle h^{\prime}(s), f(s)\right\rangle_{\mathfrak{f}} F^{\dagger}(s)+H^{\dagger}(s)\right] u \otimes \varphi(h), M^{\prime}(s) u^{\prime} \otimes \psi\left(h^{\prime}\right)\right\rangle \\
& \quad+\left\langle\Pi^{\dagger}(s) h(s) \otimes E^{\dagger}(s) u \otimes \psi(h)+f(s) \otimes E^{\dagger}(s) u \otimes \psi(h),\right.
\end{aligned}
$$




$$
\begin{aligned}
& \left.\left.\Pi^{\prime}(s) h^{\prime}(s) \otimes E^{\prime}(s) u^{\prime} \otimes \psi\left(h^{\prime}\right)+g^{\prime}(s) \otimes G^{\prime}(s) u^{\prime} \otimes \psi\left(h^{\prime}\right)\right\rangle\right\} d s \\
& =\int_{0}^{t}\left\langle u \otimes \psi(h),\left\{\left\langle h(s), \Pi^{\prime}(s) h^{\prime}(s)\right\rangle_{\mathfrak{f}} M(s) E^{\prime}(s)\right.\right. \\
& \quad+\left\langle f^{\prime}(s), h^{\prime}(s)\right\rangle_{\mathfrak{f}} M(s) F^{\prime}(s)+\left\langle h(s), g^{\prime}(s)\right\rangle_{\mathfrak{f}} M(s) G^{\prime}(s)+M(s) H^{\prime}(s) \\
& \quad+\left\langle h(s), \Pi(s) h^{\prime}(s)\right\rangle_{\mathfrak{f}} E(s) M^{\prime}(s)+\langle h(s), g(s)\rangle_{\mathfrak{f}} G(s) M^{\prime}(s) \\
& \quad+\left\langle f(s), h^{\prime}(s)\right\rangle_{\mathfrak{f}} F(s) M^{\prime}(s)+H(s) M^{\prime}(s) \\
& \quad+\left\langle h(s), \Pi(s) \Pi^{\prime}(s) h^{\prime}(s)\right\rangle_{\mathfrak{f}} E(s) E^{\prime}(s)+\langle h(s), \Pi(s) h(s)\rangle_{\mathfrak{f}} E(s) G^{\prime}(s) \\
& \left.\left.\quad+\left\langle\Pi^{\prime \dagger}(s) f(s), h^{\prime}(s)\right\rangle_{\mathfrak{f}} F(s) E^{\prime}(s)+\left\langle f(s), g^{\prime}(s)\right\rangle F(s) G^{\prime}(s)\right\} u^{\prime} \otimes \psi\left(h^{\prime}\right)\right\rangle d s .
\end{aligned}
$$

Comparing this expression with (4.4) we obtain the theorem.

\section{Reduction to the Ito-Integral for Brownian Motion}

In this section we take $\mathfrak{f}=\mathfrak{R}=\mathbb{C}$, and write $A(t)=A_{f}(t), A^{\dagger}(t)=A_{f}^{\dagger}(t)$, where $f(t) \equiv 1$. The Fock space $\widetilde{\mathfrak{H}}=\mathfrak{H}=\Gamma\left(L^{2}[0, \infty)\right)$ is identified by means of the duality transformation with $L^{2}(\Omega, w)$, where $w$ is Wiener measure on the canonical Brownian path space. We identify each random variable $\xi$ on $\Omega$ with the operator of multiplication by $\xi$; then

$$
A(t)+A^{\dagger}(t)=\omega(t)
$$

where $\omega(t)$ is the evaluation of the Brownian path $\omega$ at time $t$. We take as admissible subspace $S$ the set of bounded purely imaginary functions in $L^{2}[0, \infty)$, and write $\mathfrak{U}_{0}=\mathfrak{A}_{0}(\mathbb{C}, S), L^{2}=L^{2}(\mathbb{C}, S)$. As an element of $L^{2}(\Omega, w)$,

$$
\psi(h)(\omega)=\exp \left(\int_{0}^{\infty} h d \omega-\frac{1}{2} \int_{0}^{\infty} h^{2} d s\right),
$$

where $\int_{0}^{\infty} h d \omega$ denotes the Wiener integral.

It is well known [11] that if $\alpha$ is a nonanticipating Brownian functional for which, for every $t \geqq 0$,

$$
\int_{0}^{t} \mathbb{E}\left[\alpha(s, \cdot)^{2}\right] d s<\infty
$$

then there exists a sequence of simple nonanticipating Brownian functionals $\alpha^{(n)}, n=1,2, \ldots$ such that for all $t \geqq 0$

$$
\lim _{n} \int_{0}^{t} \mathbb{E}\left[\left(\alpha(s, \cdot)-\alpha^{(n)}(s, \cdot)\right)^{2}\right] d s=0
$$

Proposition 5.1. Let $\alpha$ be a nonanticipating Brownian functional satisfying (5.2). Then the multiplication operator valued process $\alpha=(\alpha(t, \cdot): t \geqq 0) \in L^{2}$.

Proof. Let $\alpha^{(n)}, n=1,2, \ldots$ be simple nonanticipating Brownian functionals satisfying (5.4). Then as operator valued processes the $\alpha^{(n)} \in \mathfrak{U}_{0}$. Moreover for 
arbitrary $h \in \mathfrak{h}$ and $t>0$, using (5.2) we have

$$
\begin{aligned}
\int_{0}^{t} \| & {\left[\alpha(s, \cdot)-\alpha^{(n)}(s, \cdot)\right] \psi(h) \|^{2} d s } \\
& =\int_{0}^{t} \mathbb{E}\left|\left(\alpha(s, \omega)-\alpha^{(n)}(s, \omega)\right) \exp \left(\int_{0}^{\infty} h d \omega-\frac{1}{2} \int_{0}^{\infty} h^{2}\right)\right|^{2} w(d \omega) d s \\
& =\exp \left(-\frac{1}{2} \int_{0}^{\infty} h^{2}\right) \int_{0}^{t} \mathbb{E}\left(\alpha(s, \cdot)-\alpha^{(n)}(s, \cdot)\right)^{2} d s \underset{n}{\longrightarrow} 0,
\end{aligned}
$$

since $\int_{0}^{\infty} h d \omega$ is purely imaginary and $\exp \left(-\frac{1}{2} \int_{0}^{\infty} h^{2}\right)$ is sure. Hence $\alpha \in L^{2}$.

Now let $\alpha$ and $\beta$ be nonanticipating Brownian functionals satisfying the condition (5.3), and let

$$
m(t, \omega)=\int_{0}^{t} \alpha(s, \omega) d \omega(s)+\int_{0}^{t} \beta(s, \omega) d s
$$

where the first term is the usual Ito-Doob mean-square integral of $\alpha$. Then we can write

$$
m(t, \cdot)=\int_{0}^{t}\left(\alpha(s, \cdot) d A+\alpha(s, \cdot) d A^{\dagger}+\beta(s, \cdot) d s\right) .
$$

For simple $\alpha$ this follows from (5.1); for general $\alpha$ it follows from Proposition 5.5. Suppose that $\alpha$ and $\beta$ are bounded. By Corollary 2 to Theorem 4.3, $m \in L^{2}$, and since $\alpha$ and $\beta$ are bounded, $\alpha m$ and $\beta m \in L^{2}$ also. We derive the classical Ito formula $d m^{2}=2 m d m+\alpha^{2} d t$ as follows:

Theorem 5.1. Let $\alpha$ and $\beta$ be bounded real-valued nonanticipating Brownian functionals, and let

$$
m(t, \omega)=\int_{0}^{t} \alpha d \omega+\int_{0}^{t} \beta d s
$$

Then

$$
m(t, \omega)^{2}=\int_{0}^{t} 2 m \alpha d \omega+\int_{0}^{t}\left(2 m \beta+\alpha^{2}\right) d s .
$$

Proof. In Theorem 4.3, with $S^{\prime}=S, \mathfrak{D}^{\prime}=\mathfrak{D}=\mathbb{C}, \Pi=\Pi^{\prime}=0, f^{\prime}=g^{\prime}=f=g=1$, $u=u^{\prime}=1$, take $E=E^{\prime}=0, F(t)=G(t)=F^{\prime}(t)=G^{\prime}(t)=\alpha(t, \cdot), H(t)=H^{\prime}(t)=\beta(t, \cdot)$. Then, since $h, h^{\prime}$ are imaginary valued, the left side of (4.7) is

$$
\mathbb{E}\left[m(t, \omega)^{2} \exp \left\{\int_{0}^{\infty}\left(-h+h^{\prime}\right) d \omega-\frac{1}{2} \int_{0}^{\infty}\left(h^{2}+h^{\prime 2}\right)\right\}\right],
$$

while the right side is seen to be

$$
\begin{aligned}
& \mathbb{E}\left[\int_{0}^{t}\left\{2 \alpha(s, \omega) m(s, \omega) d \omega(s)+\left(2 \beta(s, \omega) m(s, \omega)+\alpha^{2}(s, \omega)\right) d s\right\}\right. \\
& \left.\cdot \exp \left\{\int_{0}^{\infty}\left(-h+h^{\prime}\right) d \omega-\frac{1}{2} \int_{0}^{\infty}\left(h^{2}+h^{\prime 2}\right)\right\}\right] .
\end{aligned}
$$


Equating (5.7) to (5.8) and cancelling the sure factor exp $\left\{-\frac{1}{2} \int_{0}^{\infty}\left(h^{2}+h^{\prime 2}\right)\right\}$,

$$
\begin{aligned}
& \mathbb{E}\left[\left\{m(t, \omega)^{2}-\int_{0}^{t}[2 \alpha(s, \omega) m(s, \omega) d \omega(s)+(2 \beta(s, \omega) m(s, \omega)\right.\right. \\
& \left.\left.\left.\quad+\alpha^{2}(s, \omega)\right) d s\right] \exp \int_{0}^{\infty} h^{\prime \prime} d \omega\right]=0,
\end{aligned}
$$

where $h^{\prime \prime}=-h+h^{\prime}$. Since the functionals $\exp \int_{0}^{\omega} h^{\prime \prime} d \omega$ are total, (5.6) holds.

\section{The Poisson Process in Fock Space}

In this section we take $K=\mathbb{C}$ so that $\tilde{\mathfrak{H}}=\mathfrak{H}$. Let $\Pi$ be a self adjoint valued element of $L_{B(\mathfrak{f})}^{\infty}$, loc $[0, \infty)$, let $f \in L_{\mathfrak{f}}^{\infty, l^{\circ}}[0, \infty)$ and consider the unitary process

$$
W_{f, \Pi}(t)=W\left(f_{t}, e^{i \Pi_{t}}\right),
$$

where $W(f, U)$ is defined by (2.1). In view of (2.5) $W_{f, I}$ is the product $V_{1} V_{2} V_{3} V_{4}$ of the processes defined by

$$
\begin{aligned}
& V_{1}(t) \psi(h)=e^{-\frac{1}{2}\left\|f_{t}\right\|_{\mathrm{f}}^{2}} \psi(h) \\
& V_{2}(t) \psi(h)=e^{A_{f}^{\dagger}(t)} \psi(h)=\psi\left(h+f_{t}\right) \\
& V_{3}(t) \psi(h)=W\left(0, e^{i \Pi_{t}}\right) \psi(h)=\psi\left(e^{i \Pi_{t}} h\right) \\
& V_{4}(t) \psi(h)=e^{-A_{\exp (-\imath \Pi) f}(t)} \psi(h)=e^{\left.-\langle\exp (-i \Pi) f)_{t}, h_{t}\right\rangle} \psi(h) .
\end{aligned}
$$

We consider $W_{f, \Pi}$ and $V_{j}, j=1,2,3,4$ as processes based on $(\mathbb{C}, S)$, where $S$ consists of all locally bounded elements of $\mathfrak{h}$. From Theorem 4.1 we have

$$
\begin{aligned}
& d V_{1}=V_{1}\left(-\frac{1}{2}\|f(t)\|_{\mathfrak{f}}^{2}\right) d t, \\
& d V_{2}=V_{2} d A_{f}^{\dagger}, \\
& d V_{3}=V_{3} d \Lambda_{(\exp (i \Pi)-I),} \\
& d V_{4}=V_{4} d A_{\exp (-i \Pi) f} .
\end{aligned}
$$

Since $W_{f, \Pi}(t)$ is the product of these processes on $\mathfrak{\Xi}$, from use of (4.14),

$$
d W_{f, \Pi}=W_{f, \Pi}\left(d \Lambda_{\left(e^{i \Pi-I)}\right.}-d A_{e^{-i \Pi f}}+d A_{f}^{\dagger}-\frac{1}{2}\|f(t)\|_{\mathfrak{f}}^{2} d t\right) .
$$

In particular $W_{f, I}$ is the solution of a "stochastic differential equation" and $W_{f, \Pi}$ $\in \mathfrak{C}(S)$.

Now let $\mathfrak{f}=\mathbb{C}, \Pi(t)=\alpha(t), f(t)=\sqrt{l}\left(e^{i \alpha(t)}-1\right)$ and

$$
V_{\alpha}(t)=\exp \left(i \int_{0}^{t} l \sin \alpha(s) d s\right) W_{f, \Pi}(t)
$$

where $\alpha$ is a locally bounded function and $l \geqq 0$ is a constant. Then

$$
V_{\alpha}(t) \psi(h)=\exp \left(\int_{0}^{t}\left\{l\left(e^{i \alpha}-1\right)+\sqrt{l}\left(e^{i \alpha}-1\right) h\right\}\right) \psi\left(e^{i \alpha_{t}} h+\sqrt{l}\left(e^{i \alpha_{t}}-1\right)\right) .
$$


Proposition 6.2. Let $\alpha, \beta$ be real-valued locally bounded Lebesgue measurable functions on $[0, \infty)$ and let $h, h^{\prime} \in L^{2}[0, \infty)$. Then
a) $V_{\alpha}(t)$ is unitary for all $t \geqq 0$,
b) $V_{\alpha}(t) V_{\beta}(t)=V_{\alpha+\beta}(t)$,
c) $\left\langle\psi(h), V_{\alpha}(t) \psi\left(h^{\prime}\right)\right\rangle=\exp \left\{\int_{0}^{t}\left(e^{i \alpha}-1\right)(\bar{h}+\sqrt{l})\left(h^{\prime}+\sqrt{l}\right)\right\}\left\langle\psi(h), \psi\left(h^{\prime}\right)\right\rangle$,

in particular

$$
\left\langle\psi(0), V_{\alpha}(t) \psi(0)\right\rangle=\exp \left\{l \int_{0}^{t}\left(e^{i \alpha}-1\right)\right\}
$$

d) $\left\{V_{\alpha}(t) \psi(0), \alpha \in L_{\mathbb{R}}^{\infty,{ }^{\text {loc }}}[0, \infty)\right\}$ is total in $\mathfrak{H}_{t}$ for all $t \geqq 0$.

Proof. a) and b) follow from (6.2) and (2.2) and c) follows from (6.3). To prove d) note that for arbitrary real numbers $\theta, \alpha_{1}, \alpha_{2}, \ldots, \alpha_{N}$, and disjoint Borel sets $S_{1}, S_{2}, \ldots, S_{N}$, the linear span of the $V(t) \psi_{N}(0)$ contains the vector

$$
\psi\left(\sqrt{l} \sum_{j=1}^{N}\left(e^{i \theta \alpha_{J}}-1\right) \chi_{s_{j} \cap[0, t]}\right),
$$

obtained by taking $\alpha=\theta \sum_{j=1}^{N} \alpha_{j} \chi_{s_{j}}$. Hence, for $n=0,1,2, \ldots$, the closure of the linear span contains the vector

$$
\left.\left(-i \frac{d}{d \theta}\right)^{n} \psi\left(\sqrt{l}\left(e^{i \theta \alpha_{j}}-1\right) \chi_{S_{j} \cap[0, t]}\right)\right|_{\theta=0}
$$

whose expansion in $n$-particle components is of form

$$
(*, *, \ldots, \sqrt{n !} f \otimes \ldots \otimes f, 0,0, \ldots),
$$

where $f=\sum_{j=1}^{N} \alpha_{j} \chi_{S_{j} \cap[0, t]}$. Since such vectors are total in $\mathfrak{H}_{t}=\Gamma\left(L^{2}[0, t]\right)$, the result follows.

We recognise the vacuum expectation functional (6.5) as the characteristic functional

$$
\mathbb{E} \exp \left(i \int_{0}^{t} \alpha d X_{l}\right)=\exp \left(l \int_{0}^{t}\left(e^{i \alpha}-1\right)\right)
$$

of the classical stochastic integral of $\alpha$ against the Poisson process of intensity $l$ [9]. We compare this with the identity

$$
\left\langle\psi(0), \exp \left(A_{f}^{\dagger}(t)-A_{f}(t)\right) \psi(0)\right\rangle=\exp \left(-\frac{1}{2} \int_{0}^{t} f^{2}\right)=\mathbb{E} \exp \left(i \int_{0}^{t} f d X\right),
$$

where $X$ is standard Brownian motion. Using Proposition 6.1d) and the uniqueness of the GNS construction we obtain the following analog of the duality transformation for Brownian motion. 
Theorem 6.1. There exists a unique Hilbert space isomorphism $D_{l}$ from the Fock space $\Gamma\left(L^{2}[0, \infty)\right)$ to $L^{2}\left(\mu_{l}\right)$, where $\mu_{l}$ is the probability measure for the Poisson process $X_{l}$ of intensity $l$, satisfying the conditions

a) $D_{l} \psi(0) \equiv 1$ bounded measurable function $\alpha$ on $[0, \infty)$.

Furthermore for arbitrary $t \geqq 0$ the image of $\Gamma\left(L^{2}[0, t)\right)$ under $D_{l}$ is the subspace of $L^{2}\left(\mu_{l}\right)$ of elements measurable with respect to the $\sigma$-algebra generated by $X_{l}(s)$, $s \leqq t$.

We may thus construct an operator-valued realisation of the Poisson process of intensity $l$, corresponding to the realisation $A(t)+A^{\dagger}(t)$ of Brownian motion, by defining

$$
\Pi_{l}(t)=-\left.i \frac{d}{d \varepsilon} V_{\varepsilon}(t)\right|_{\varepsilon=0},
$$

where the suffix $\varepsilon$ denotes the constant function of value $\varepsilon$. The family of self adjoint operators $\left(\Pi_{l}(t): t \geqq 0\right)$ is clearly commutative in the sense that the one parameter groups of which they are the infinitesimal generators commute; equivalently their spectral projectors commute. Under conjugation by the isomorphism $D_{l}$ the $\Pi_{l}(t)$ are simultaneously diagonalised, transforming into the operators of multiplication by the $X_{l}(t)$.

Theorem 6.2. $\Pi_{l}$ satisfies

$$
\Pi_{l}(0)=0, \quad d \Pi_{l}=d \Lambda+\sqrt{l}\left(d A+d A^{\dagger}\right)+l d t,
$$

where $\Lambda=\Lambda_{I I}, A=A_{f}, A^{\dagger}=A_{f}^{\dagger}$, with $\Pi \equiv f \equiv 1$.

Proof. The theorem is a consequence of Theorem 4.1, together with the identity, which follows from (6.6) and (6.4),

$$
\left\langle\psi(h), \Pi_{l}(t) \psi\left(h^{\prime}\right)\right\rangle=\int_{0}^{t}\left\langle\psi(h),\left\{\bar{h}(s) h^{\prime}(s)+\sqrt{l}\left(\bar{h}(s)+h^{\prime}(s)\right)+l\right\} \psi\left(h^{\prime}\right)\right\rangle d s .
$$

As immediate corollaries we obtain, using (4.14), the well-known formula for the Poisson process $\left(d \Pi_{l}\right)^{2}=d \Pi_{l}$, and, rearranging the integrated form of the Theorem as

$$
l^{-1 / 2}\left(\Pi_{l}(t)-l t\right)=A(t)+A^{\dagger}(t)+l^{-1 / 2} \Lambda,
$$

a form of the well known central limit theorem for Poisson processes of increasing intensities that $l^{-1 / 2}\left(\Pi_{l}(t)-l t\right)$ approaches Brownian motion, in which the gauge process $\Lambda$ measures the error in the Brownian approximation. Note that no two of the processes in (6.7) commute.

Using (4.14) it is easily seen that

$$
d\left[\Pi_{l}, \Pi_{n}\right]=(\sqrt{l}-\sqrt{m})\left(d A-d A^{\dagger}\right),
$$

so that

$$
\left[\Pi_{l}, \Pi_{m}\right]=(\sqrt{l}-\sqrt{m})\left(A-A^{\dagger}\right)
$$


In particular the Poisson processes of different intensities do not commute with each other and may not be simultaneously diagonalised; for $l \neq m, D_{l} \neq D_{m}$.

We end this section by noting that, by generalising these methods, a Fock space realisation of an arbitrary classical stochastic process with independent increments can be obtained whose differential is expressible in terms of appropriate creation, annihilation and gauge processes using (4.12). Details will be published elsewhere.

\section{Stochastic Evolutions}

In this section, for simplicity, $\mathfrak{f}=\mathbb{C}$, but $\Omega$ is arbitrary. For the three basic processes $A, A, A^{\dagger}$ defined by

$$
\Lambda(t)=\Lambda_{\Pi I}(t), \quad A(t)=A_{f}(t), \quad A^{\dagger}(t)=A_{f}^{\dagger}(t)
$$

with $\Pi(t) \equiv f(t) \equiv 1,(4.14)$ becomes

\begin{tabular}{c|cccc} 
& $d \Lambda$ & $d A$ & $d A^{\dagger}$ & $d t$ \\
\hline$d \Lambda$ & $d \Lambda$ & 0 & $d A^{\dagger}$ & 0 \\
$d A$ & $d A$ & 0 & $d t$ & 0 \\
$d A^{\dagger}$ & 0 & 0 & 0 & 0 \\
$d t$ & 0 & 0 & 0 & 0
\end{tabular}

Let $L_{j}, j=1,2,3,4$ be operators in the initial space $\mathfrak{R}$ with common invariant domain $\mathfrak{D}$; we regard them also as operators in $\mathfrak{H}$ with domain $\mathfrak{D} \otimes \mathfrak{G}$. Our aim is to construct an operator-valued process $U$ satisfying

$$
U(0)=I, \quad d U=U\left(L_{1} d \Lambda+L_{2} d A+L_{3} d A^{\dagger}+L_{4} d t\right)
$$

and to find conditions on $L_{j}, j=1,2,3,4$ ensuring unitarity of $U$.

We construct such stochastic evolutions by iteration. We take the admissible subspace $S$ to consist of all locally bounded functions in $L^{2}[0, \infty)$.

Proposition 7.1. There exist processes $U_{n}, n=0,1,2, \ldots$, satisfying

$$
U_{0}(t)=I, \quad U_{n}(t)=I+\int_{0}^{t} U_{n-1}\left(L_{1} d \Lambda+L_{2} d A+L_{3} d A^{\dagger}+L_{4} d s\right) .
$$

Furthermore for arbitrary $u \in \mathfrak{D}, h \in S, T>0$ and $0 \leqq t \leqq T$,

$$
\left\|\left(U_{n}(t)-U_{n-1}(t)\right) u \otimes \psi(h)\right\|^{2} \leqq e^{T+\|h\|^{2}}(n !)^{-1} \beta(T)^{n} \max _{1 \leqq j_{1}, \ldots, j_{n} \leqq 4}\left\|L_{j_{1}} \cdots L_{j_{n}} u\right\|^{2},
$$

where $\beta(T)=24 T \max \left(1, \sup _{0 \leqq s \leqq T}|h(s)|^{4}\right)$.

Proof. $U_{0}$ is clearly in $L^{2}(\mathfrak{D}, S)$. Suppose that $U_{n-1} \in L^{2}(\mathfrak{D}, S)$, so that for all $u \in \mathfrak{D}$ and $h \in S$,

$$
\int_{0}^{t}\left\|U_{n-1}(s) u \otimes \psi(h)\right\|^{2} d s<\infty .
$$


Since $\mathfrak{D}$ is invariant under the $L_{j}$

$\left.\int_{0}^{t} \sum_{j=1}^{4} \| U_{n-1}(s) L_{j}\right) u \otimes \psi(h)\left\|^{2} d s=\int_{0}^{t} \sum_{j=1}^{4}\right\| U_{n-1}(s)\left(L_{j} u\right) \otimes \psi(h) \|^{2} d s<\infty$,

hence $U_{n}$ is well defined and by Corollary 2 to Theorem $4.3 U_{n} \in L^{2}(\mathfrak{D}, S)$.

To prove (7.4) we write

$$
\begin{aligned}
& \left(U_{n}(t)-U_{n-1}(t)\right) u \otimes \psi(h) \\
& \quad=\int_{0}^{t}\left(U_{n-1}(t)-U_{n-2}(t)\right)\left(L_{1} d \Lambda+L_{2} d A+L_{3} d A^{\dagger}+L_{4} d s\right) u \otimes \psi(h),
\end{aligned}
$$

and apply Corollary 1 to Theorem 4.3 to obtain

$$
\begin{aligned}
& \left\|\left(U_{n}(t)-U_{n-1}(t)\right) u \otimes \psi(h)\right\|^{2} \\
& \quad \leqq 6 \alpha(T)^{2} \int_{0}^{t} e^{t-s} \sum_{j=1}^{4}\left\|\left(U_{n-1}(s)-U_{n-2}(s)\right) L_{j} u \otimes \psi(h)\right\|^{2} d s
\end{aligned}
$$

for all $t \in[0, T]$, where $\alpha(T)=\sup _{0 \leqq s \leqq T} \max \left(|h(s)|^{2},|h(s)|, 1\right)$. From this,

$$
\begin{aligned}
& \left\|\left(U_{n}(t)-U_{n-1}(t)\right) u \otimes \psi(h)\right\|^{2} \\
& \quad \leqq 24 \alpha(T)^{2} e^{t} \int_{0}^{t} e^{-s} \max _{1 \leqq j \leqq 4}\left\|\left(U_{n-1}(s)-U_{n-2}(s)\right) L_{j} u \otimes \psi(h)\right\|^{2} d s
\end{aligned}
$$

from which (7.4) follows by induction.

For $n=0,1,2, \ldots$, and $u \in \mathfrak{D}$, let

$$
M_{n}(u)=\max \left\{\left\|L_{j_{1}} \ldots L_{j_{n}} u\right\|: 1 \leqq j_{1}, \ldots, j_{n} \leqq 4\right\}
$$

and

$$
\mathfrak{D}_{0}=\left\{u \in \mathfrak{D}: \sum_{n=0}^{\infty} \varrho^{n}(n !)^{-1 / 2} M_{n}(u)<\infty \text { for all } \varrho>0\right\} .
$$

$\mathfrak{D}_{0}$ is easily seen to be invariant under each $L_{j}, 1 \leqq j \leqq 4$. Using (7.5), for $u \in \mathfrak{D}_{0}$, and arbitrary $h \in S$ and $T>0$,

$$
\sum_{n=1}^{\infty} \sup _{0 \leqq t \leqq T}\left\|\left(U_{n}(t)-U_{n-1}(t)\right) u \otimes \psi(h)\right\| \leqq e^{\frac{1}{2}\left(T+\|h\|^{2}\right)} \sum_{n=1}^{\infty}(n !)^{-1 / 2} \beta(T)^{n-2} M_{n}(u)<\infty,
$$

showing that $U_{n}(t) u \otimes \psi(h)$ converges in $\widetilde{\mathfrak{H}}$ uniformly in each finite interval $[0, T]$. The limit is clearly linear in $u$ and so defines an operator in $\widetilde{\mathfrak{H}}$ with domain $\mathfrak{D}_{0} \otimes \mathfrak{S}$. Being the strong limit on this domain of ampliations of operators in $\widetilde{\mathfrak{H}}_{t}$ with domains $\mathfrak{D} \otimes \mathfrak{\Xi}_{t}, U(t)$ extends to the ampliation of an operator in $\widetilde{\mathfrak{H}}_{t}$ with domain $\mathfrak{D}_{0} \otimes \mathfrak{\Xi}_{t}$ to $\mathfrak{D}_{0} \otimes \mathfrak{\Xi}_{t} \otimes \mathfrak{S}^{t}$. Thus extended, the $U(t)$ form an adapted process $U$. Using the uniformity of the convergence together with the estimate (7.4), we may take strong limits on $\mathfrak{D}_{0} \otimes \mathbb{S}$ on both sides of (7.3) to conclude that $U$ satisfies (7.2) and belongs to $\mathfrak{U}_{c}\left(\mathfrak{D}_{0}, S\right)$. In particular $U \in L^{2}\left(\mathfrak{D}_{0}, S\right)$.

Suppose $V \in L^{2}\left(\mathfrak{D}_{0}, S\right)$ is another process satisfying (7.2). Then $U(0)-V(0)=0$ and by the argument employed in proving (7.4), we have

$$
\|(U(t)-V(t)) u \otimes \psi(h)\|^{2} \leqq e^{T+\|h\|^{2}}(n !)^{-1} \beta(T)^{n}\left(M_{n}(u)\right)^{2}
$$


for all $t \in[0, T]$. Letting $n \rightarrow \infty$ on the right side we conclude that $U(t)$ and $V(t)$ agree on $\mathfrak{D}_{0} \otimes \mathfrak{S}$. Thus the solution of $(7.2)$ is unique as a process in $L^{2}\left(\mathfrak{D}_{0}, S\right)$.

We now assume that the operators $L_{j}$ are bounded with domain $\mathfrak{R}$ so that $\mathfrak{D}_{0}=\Re$ also. We investigate when the process $U$ is unitary. If this is the case then, using (7.1) and

$$
\begin{gathered}
d U=U\left(L_{1} d \Lambda+L_{2} d A+L_{3} d A^{\dagger}+L_{4} d t\right), \\
d U^{\dagger}=L_{1}^{\dagger} U^{\dagger} d \Lambda+L_{3}^{\dagger} U^{\dagger} d A+L_{2}^{\dagger} U^{\dagger} d A^{\dagger}+L_{4}^{\dagger} U^{\dagger} d t
\end{gathered}
$$

together with the unitary condition $U^{\dagger} U=I$, we have

$$
\begin{aligned}
0= & d\left(U^{\dagger} U\right)=\left(d U^{\dagger}\right) U+U^{\dagger} d U+d U^{\dagger} d U \\
= & \left(L_{1}^{\dagger} d \Lambda+L_{3}^{\dagger} d A+L_{2}^{\dagger} d A^{\dagger}+L_{4}^{\dagger} d t\right)+\left(L_{1} d \Lambda+L_{2} d A+L_{3} d A^{\dagger}+L_{4} d t\right) \\
& +\left(L_{1}^{\dagger} L_{1} d \Lambda+L_{1}^{\dagger} L_{3} d A^{\dagger}+L_{3}^{\dagger} L_{1} d A+L_{3}^{\dagger} L_{3} d t\right) \\
= & \left(L_{1}^{\dagger}+L_{1}+L_{1}^{\dagger} L_{1}\right) d \Lambda+\left(L_{2}+L_{3}^{\dagger}+L_{3}^{\dagger} L_{1}\right) d A+\left(L_{3}+L_{2}^{\dagger}+L_{1}^{\dagger} L_{3}\right) d A^{\dagger} \\
& +\left(L_{4}+L_{4}^{\dagger}+L_{3}^{\dagger} L_{3}\right) d t .
\end{aligned}
$$

In view of Theorem 4.1 we may equate coefficients of the differentials to zero, obtaining

$$
\begin{aligned}
& L_{1}+L_{1}^{\dagger}+L_{1}^{\dagger} L_{1}=0, \\
& L_{2}+L_{3}^{\dagger}+L_{3}^{\dagger} L_{1}=0, \\
& L_{3}+L_{2}^{\dagger}+L_{1}^{\dagger} L_{3}=0, \\
& L_{4}+L_{4}^{\dagger}+L_{3}^{\dagger} L_{3}=0 .
\end{aligned}
$$

Similarly, from $U U^{\dagger}=I$ we obtain

$$
0=d\left(U U^{\dagger}\right)=U\left(L_{1}+L_{1}^{\dagger}+L_{1} L_{1}^{\dagger}\right) U^{\dagger} d \Lambda+\text { terms in } d A, d A^{\dagger} \text {, and } d t,
$$

and hence, multiplying on the left by $U^{\dagger}$ and on the right by $U$,

$$
L_{1}+L_{1}^{\dagger}+L_{1} L_{1}^{\dagger}=0 \text {. }
$$

Setting $W=I+L_{1},(7.5)$ and (7.9) imply that $W$ is unitary. We set $L=L_{2}$, then (7.7) implies that $L_{3}=-W L^{\dagger}$, and (7.8) that $L_{4}=i H-\frac{1}{2} L L^{\dagger}$, where $H$ is a bounded self adjoint operator. Thus for unitarity of $U$ it is necessary that

$$
\left(L_{1}, L_{2}, L_{3}, L_{4}\right)=\left(W-I, L,-W L^{\dagger}, i H-\frac{1}{2} L L^{\dagger}\right),
$$

where $W, L, H \in B(\Re), W$ is unitary and $H$ is self adjoint. Remarkably this condition is also sufficient.

Theorem 7.1. If the condition (7.10) holds, then the unique solution of (7.2) in $\mathfrak{A}_{c}(\mathfrak{N}, S)$ is a unitary process. In particular $U \in \mathfrak{C}(S)$.

Proof. From (7.4), for $u \in \mathfrak{K}, h \in S, T>0$, and $0 \leqq t \leqq T$,

$$
\|U(t) u \otimes \psi(h)\| \leqq\|u \otimes \psi(h)\|+\sum_{n=1}^{\infty}\left\|\left(U_{n}(t)-U_{n-1}(t)\right) u \otimes \psi(h)\right\| \leqq m_{T, h}\|u\|,
$$

where

$$
m_{T, h}=e^{-\frac{1}{2}\|h\|^{2}} \sum_{n=0}^{\infty}(n !)^{-1 / 2}\left(\beta(\mathrm{T})^{1 / 2} \lambda\right)^{n}, \quad \lambda=\max \left(\|L\|,\left\|i H-\frac{1}{2} L L\right\|,\|W-I\|\right) .
$$


It follows from (7.11) that for fixed $h, h^{\prime} \in S$, there exist operators $K(t), t \geqq 0$ in $B(\Omega)$ for which, for arbitrary $u, v \in \mathfrak{R}$,

$$
\langle u, K(t) v\rangle=\left\langle U(t) u \otimes \psi(h), U(t) v \otimes \psi\left(h^{\prime}\right)\right\rangle .
$$

Moreover the map $t \mapsto K(t)$ is locally bounded. From Theorem 4.3 we find that $K(\cdot)$ satisfies the differential equation

$$
\begin{aligned}
\frac{d K}{d t}= & \overline{h(t)} h^{\prime}(t)\left(W^{\dagger} K W-K\right)+\bar{h}(t)\left(L^{\dagger} K-W^{\dagger} K W L^{\dagger}\right)+h(t)\left(K L-L W^{\dagger} K W\right) \\
& +\left(i[K, H]-\frac{1}{2} K L L+L W K W L-\frac{1}{2} L L K\right),
\end{aligned}
$$

which is of form $\frac{d K}{d t}=\mathscr{L}(t) K$, where $\mathscr{L}(t): B(\boldsymbol{\Omega}) \rightarrow B(\boldsymbol{\Omega})$ is a bounded linear map. Since $K(t) \equiv\left\langle\psi(h), \psi\left(h^{\prime}\right)\right\rangle I$ satisfies this equation with the correct initial value, we conclude that $K(t)$ is indeed of this form. But then it follows from (7.12) that $U(t)$ is isometric, and in particular bounded. Hence we can use (7.1) as in the proof of the necessity of $(7.10)$ to conclude that $d\left(U U^{\dagger}\right)=0$. Hence $U U^{\dagger} \equiv U(0) U^{\dagger}(0)=I$ and the proof is complete.

\section{Stochastic Dilations of Completely Positive Semigroups and Non-Commutative Feynman-Kac Formulae}

Let $U$ be the unitary process generated by bounded operators $W, L, H \in B(\Omega)$ with $W$ unitary and $H$ self adjoint in accordance with Theorem 7.1. We define families of operators $\left(T_{t}: t \geqq 0\right)$ on $\mathcal{R}$ and $\left(\mathscr{T}_{t}: t \geqq 0\right)$ on $B(\mathcal{R})$ by

$$
T_{t}=\mathbb{E}_{0}(U(t)), \quad \mathscr{T}_{t}(X)=\mathbb{E}_{0}\left(U(t) X U(t)^{-1}\right), \quad X \in B(\Re),
$$

where the vacuum conditional expectation map $\mathbb{E}_{0}: B(\tilde{\mathfrak{H}}) \rightarrow B(\Re)$ is defined by

$$
\left\langle u, \mathbb{E}_{0}(J) v\right\rangle=\langle u \otimes \psi(0), J v \otimes \psi(0)\rangle, \quad u, v \in \mathfrak{R}, J \in B(\mathfrak{R}) .
$$

Theorem 8.1. a) $\left(T_{t}: t \geqq 0\right)$ is a uniformly continuous contraction semigroup on $\mathfrak{R}$ with infinitesimal generator

$$
\left.\frac{d}{d t} T_{t}\right|_{t=0}=i H-\frac{1}{2} L L^{\dagger} .
$$

b) $\left(\mathscr{T}_{t}: t \geqq 0\right)$ is a uniformly continuous semigroup of completely positive maps on $B(\mathfrak{R})$ whose infinitesimal generator $\mathscr{L}$ is given by

$$
\mathscr{L}(X)=i[H, X]-\frac{1}{2}\left(L L^{\dagger} X-2 L X L^{\dagger}+X L L^{\dagger}\right), \quad X \in B(\Omega) .
$$

Proof. a) $T_{t}$ is clearly a contraction. By Theorem 4.1 with $h=h^{\prime}=0, M=U-I$,

$$
\begin{aligned}
\left\langle u,\left(T_{t}-I\right) u^{\prime}\right\rangle & =\int_{0}^{t}\left\langle u \otimes \psi(0), U(s)\left(i H-\frac{1}{2} L L^{\dagger}\right) u^{\prime} \otimes \psi(0)\right\rangle d s \\
& =\int_{0}^{t}\left\langle u, T_{s}\left(i H-\frac{1}{2} L L^{\dagger}\right) u^{\prime}\right\rangle d s
\end{aligned}
$$

for arbitrary $u, u^{\prime} \in \mathfrak{R}$, from which it follows that $T_{t}=\exp t\left(i H-\frac{1}{2} L L^{\dagger}\right)$. 
b) For $X \in B(\Re)$ write $\tilde{X}(t)=U(t) X U(t)^{-1}, t \geqq 0$, so that $\mathscr{T}_{t}(X)=\mathbb{E}_{0}(\tilde{X}(t))$. Then by (7.1),

$$
\begin{aligned}
d \tilde{X}= & \left(W X W^{\dagger}-X\right)^{\tilde{d}} d \Lambda+[\tilde{L}, \tilde{X}] d A-\tilde{W}\left[\tilde{L}^{\dagger}, \tilde{X}\right] d A^{\dagger} \\
& +\left\{i[\tilde{H}, \tilde{X}]-\frac{1}{2}\left(\tilde{L} \tilde{L}^{\dagger} \tilde{X}-2 \tilde{L} \tilde{X} \tilde{L}^{\dagger}+\tilde{X} \tilde{L}^{\dagger}\right)\right\} d t
\end{aligned}
$$

Using Theorem 4.1 with $h=h^{\prime}=0$ we obtain, for $u, u^{\prime} \in \mathfrak{R}$,

$$
\left\langle u,\left(\mathscr{T}_{t}(X)-X\right) u^{\prime}\right\rangle=\int_{0}^{t}\left\langle u, \mathscr{T}_{s}(\mathscr{L}(X)) u^{\prime}\right\rangle d s
$$

from which $\mathscr{T}_{t}=e^{t \mathscr{L}} . \mathscr{T}_{t}$ is completely positive being the composition of a unitary conjugation with a conditional expectation.

We compare the generator (8.2) with the general form [10]

$$
\begin{gathered}
\mathscr{L}(X)=i[H, X]-\frac{1}{2} \sum_{j}\left(L_{j} L_{j}^{\dagger} X-2 L_{j} X L_{j}^{\dagger}+X L_{j} L_{j}^{\dagger}\right), \\
H, L_{j} \in B(\Re), \sum_{j} L_{j} L_{j}^{\dagger}<\infty
\end{gathered}
$$

of infinitesimal generator of a uniformly continuous completely positive semigroup on $B(\mathcal{R})$. Provided that there are only finitely many $L_{j}$ a stochastic dilation the semigroup generated by (8.3) can be constructed as follows; take $f_{j}(t) \equiv e_{j}, e_{j}$ being the $j^{\text {th }}$ element of the natural basis of $\mathfrak{l}=\mathbb{C}^{n}, A_{j}(t)=A_{f_{j}}(t), A_{j}^{\dagger}(t)=A_{f_{j}}^{\dagger}(t)$. Then the equation

$$
U(0)=I, \quad d U=U\left(\sum_{j}\left\{L_{j} d A-L_{j}^{\dagger} d A^{\dagger}\right\}+\left(i H-\frac{1}{2} \sum_{j} L_{j} L_{j}^{\dagger}\right) d t\right)
$$

has a unique solution in $\mathfrak{M}(\mathfrak{R}, S)$, where $S$ is all bounded elements of $\mathfrak{h}$, which is a unitary process for which the maps $\mathscr{T}_{t}$ given by $(8.1)$ form a completely positive semigroup of which (8.3) is the generator. The case of infinitely many $L_{j}$ can also be treated by an extension of the stochastic calculus developed here [8].

The gauge generator $W-I$ does not appear in the form (8.2); thus these dilations are nonunique.

We consider perturbations of the semigroups $\left(T_{t}\right)$ and $\left(\mathscr{T}_{t}\right)$. Let $V$ be fixed in $B(\mathfrak{K})$. Then there exists a unique map $t \rightarrow C(t)$ from $[0, \infty)$ into $B(\widetilde{\mathfrak{H}})$ satisfying

$$
\frac{d C}{d t}=-C U(t) V U(t)^{-1}, \quad C(0)=I,
$$

where differentiation is in the uniform sense; furthermore $C(t)$ has a bounded inverse for all $t \geqq 0$ and

$$
\frac{d C^{-1}}{d t}=U(t) V U(t)^{-1} C^{-1} .
$$

Define operators $\hat{U}(t)=C(t) U(t), t \geqq 0$. Then $\hat{U} \in \mathfrak{M}(S)$ and satisfies

$$
d \hat{U}=\hat{U}\left(L d A-L^{\dagger} d A^{\dagger}+\left(i H-\frac{1}{2} L L^{\dagger}-V\right) d t\right) .
$$

Repetition of the proof of Theorem 8.1a) shows that $T_{t}=\mathbb{E}_{0}(U(t)), t \geqq 0$, defines a one parameter semigroup of bounded operators on $\boldsymbol{R}$ of which the infinitesimal 
generator is $i H-\frac{1}{2} L L^{\dagger}-V$. Thus

$$
\exp \left(t\left(i H-\frac{1}{2} L L^{\dagger}-V\right)\right)=\mathbb{E}_{0}(C(t) U(t)) .
$$

When the operators $\tilde{V}(t)=U(t) V U(t)^{-1}$ commute, $C(t)$ can be expressed as $\exp \left(-\int_{0}^{t} \tilde{V}(s) d s\right)$ and (8.6) takes the form of a Feynman-Kac formula as in [5].

Similarly, the formula $\hat{\mathscr{T}}_{t}(X)=\mathbb{E}_{0}\left[\hat{U}(t) X \hat{U}(t)^{-1}\right]$ defines a uniformly continuous semigroup on $B(\Re)$ with infinitesimal generator $\hat{\mathscr{L}}$, where $\hat{\mathscr{L}}(X)=\mathscr{L}(X)$ $-[V, X]$. Thus

$$
\exp \{t(\mathscr{L}-\operatorname{ad} V)\}(X)=\mathbb{E}_{0}\left(C(t) U(t) X U(t)^{-1} C(t)^{-1}\right),
$$

where ad $V(X)=[V, X]$. Equation (8.7) is a Feynman-Kac formula in the sense of [1].

There exist examples where (7.2) admits a unitary solution for which analogs of Theorem 8.1 and (8.6) and (8.7) hold in which $L, H$, and $V$ are unbounded operators. We defer discussion to a later article.

\section{References}

1. Accardi, L.: On the quantum Feynman-Kac formula. Rend. Sem. Mat. Fis. Milano 48, 135-180 (1980)

2. Applebaum, D., Hudson, R.L.: Fermion diffusions. J. Math. Phys. (to appear)

3. Barnett, C., Steater, R.F., Wilde, I.: The Ito-Clifford integral. J. Funct. Anal. 48, 172-212 (1982)

4. Gorini, V., Kossakowski, A., Sudarshan, E.C.G.: Completely positive dynamical semigroups of $n$-level systems. J. Math. Phys. 17, 821-5 (1976)

5. Hudson, R.L., Ion, P.D.F., Parthasarathy, K.R.: Time-orthogonal unitary dilations and noncommutative Feynman-Kac formulae. Commun. Math. Phys. 83, 261-80 (1982)

6. Hudson, R.L., Karandikar, R.L., Parthasarathy, K.R.: Towards a theory of noncommutative semimartingales adapted to Brownian motion and a quantum Ito's formula; and Hudson, R.L., Parthasarathy, K.R.: Quantum diffusions. In: Theory and applications of random fields, Kallianpur, (ed.). Lecture Notes in Control Theory and Information Sciences 49, Berlin, Heidelberg, New York, Tokyo: Springer 1983

7. Hudson, R.L., Parthasarathy, K.R.: Construction of quantum diffusions. In: Quantum probability and applications to the quantum theory of irreversible processes, Accardi (ed.) (to appear)

8. Hudson, R.L., Parthasarathy, K.R.: Stochastic dilations of uniformly continuous completely positive semigroups. Acta Math. Applicandae (to appear)

9. Ikeda, N., Watenabe, S.: Stochastic differential equations and diffusion processes. Amsterdam: North-Holland 1981

10. Lindblad, G.: On the generators of quantum dynamical semigroups. Commun. Math. Phys. 48, 119-30 (1976)

11. Liptser, R.S., Shiraev, A.N.: Statistics of random processes. I. General theory. Berlin, Heidelberg, New York: Springer 1977

Communicated by H. Araki

Received June 28, 1983; in revised form December 5, 1983 
